\title{
New relativistic and nonrelativistic model of diatomic molecules and fermionic particles interacting with improved modified Mobius potential in the framework of noncommutative quantum mechanics symmetries
}

\author{
Abdelmadjid Maireche ${ }^{1}$ (i) $^{\text {a }}$ \\ 1 Laboratory of Physics and Material Chemistry, Physics department, Sciences Faculty, University of M'sila-Algeria \\ Keywords: noncommutative geometry, diatomic molecules, modified mobius square potential, schrödinger equation, dirac equation, klein-gordon \\ equation, Bopp's shift method and star products \\ https://doi.org/10.53370/001c.28090
}

\section{Yanbu Journal of Engineering and Science}

In this study, the deformed Klein-Gordon equation, Dirac equation, and Schrödinger equations were solved with the improved the modified Mobius square potential model (IMSPM, in short) using Bopp's shift and standard perturbation theory methods in the symmetries of extended quantum mechanics. By employing the improved approximation to the centrifugal term, the relativistic and nonrelativistic bound state energies were obtained for some selected diatomic molecules $\left(\mathrm{H}_{2}, \mathrm{I}_{2}, \mathrm{CO}, \mathrm{NO}\right.$, and $\left.\mathrm{HCl}\right)$. The relativistic shift energy $\Delta E_{m s p}^{\text {tot }}\left(n, \alpha, A, B, V_{0}, \Theta, \sigma, \chi, j, l, s, m\right)$ and the perturbative nonrelativistic corrections $\Delta E_{m s p}^{n r}\left(n, \alpha, A, B, V_{0}, \Theta, \sigma, \chi, j, l, s, m\right)$ appeared as a function of the parameters $\left(\alpha, A, B, V_{0}\right)$, the parameters of noncommutativity $(\Theta, \sigma, \chi)$, in addition to the atomic quantum numbers $(n, j, l, s, m)$. In both relativistic and nonrelativistic problems, we show that the corrections on the spectrum energy are smaller than the main energy in the ordinary cases of RQM and NRQM. A straightforward limit of our results to ordinary quantum mechanics shows that the present result under the improved modified Mobius square potential model is consistent with what is obtained in the literature. In the new symmetries of NCQM, is not possible to get the exact analytical solutions for $l=0$ and $l \neq 0$ can only be solved approximately. Through this research, we came to two noteworthy results, the first is related to the deformed Klein-Gordon equation under the influence of the improved modified Mobius square potential model becomes equivalent to the Duffin-Kemmer equation for a meson with spin-s, while the second result concerns the deformed Schrödinger equation, which can now describe the state of high-energy fermionic particles similar to the Dirac equation in the literature.

\begin{abstract}
Abbreviations
IMSPM (Improved the modified Mobius square potential model); RQM (Relativistic quantum mechanics); NRQM (Nonrelativistic quantum mechanics); NCQM (Noncommutative quantum mechanics); NRNCQM (Nonrelativistic noncommutative quantum mechanics); DDE (deformed Dirac equation); DRKGT (deformed relativistic Klien-Gordon theory).
\end{abstract}

\section{INTRODUCTION}

Despite the passage of nearly a century on the three funda- mental equations are known in the literature Schrödinger, Klien-Gordon, and Dirac equations, they are still attracting the interest of many researchers. Finding solutions to these equations guarantees the provision of all physical and chemical information on the studied system. Systems are described by different potentials. Among these potentials is a model, the modified Mobius square potential (MMSP), which has the form $V_{m s p}(r)=-V_{0}\left(\frac{A+B e^{-2 u r}}{1-e^{-2 a r}}\right)^{2}$. This potential is one of the types of exponential potential, it received the attention of many researchers in both nonrelativistic and relativistic cases. Okorie et al. studied the bound state solutions of the Schrödinger equation with MMSP and its thermodynamic properties. ${ }^{1}$ Onyenegecha et al. obtained analytical solutions of D-dimensional Klein-Gordon equation with MMSP using the Nikiforov-Uvarov (NU) method 
and obtained the relativistic energy eigenvalues and corresponding wave functions. ${ }^{2}$ Using the parametric $\mathrm{NU}$ method, Ikot et al. $^{3}$ solved the Dirac equation for MMSP within the Yukawa-like tensor interaction. Using the newly proposed NU-functional analysis approach, Ikot et al., solved the nonrelativistic Schrödinger equation for MMSP. Ukewuihe et al. solved the Schrödinger equation in $\mathrm{D}$ dimensions with the MMSP plus Hulthén potential and obtained the energy eigenvalues and corresponding wave functions using the Nikiforov-Uvarov (NU) method. ${ }^{5}$ Very recently, Uduakobong et al. presented the bound and scattering states solutions of the Klein-Gordon equation with generalized Mobius square potential in D-dimensions. ${ }^{6}$ Okon et al. studied thermodynamic properties and bound state solutions of Schrödinger equation with Mobius square plus screened-Kratzer potential using Nikiforov-Uvarov method for two diatomic molecules $\mathrm{CO}$ and ScF. ${ }^{7}$ Okorie et $a l .{ }^{8}$ examined the diatomic molecules energy spectra for the generalized Mobius square potential model by employing the dissociation energy and equilibrium bond length as explicit parameters. The Mobius square potential is the general case of some potentials and plays a vital role in many fields, it has been used to model internuclear interaction potential curves for different states of diatomic molecules and can be used to model the internuclear interaction potential curve for different electronic states of $K 2\left(a^{3} \Sigma^{+}\right)$, $N a k\left(c^{3} \Sigma^{+}\right), K R b\left(B^{1} \prod\right), S c I\left(B^{1} \Pi\right)$ and $N I\left(c^{3} \Sigma^{+}\right) \mathrm{di}^{-}$ atomic molecules. ${ }^{8}$ Ikot et al. ${ }^{9}$ obtained solutions of the Dirac equation with Mobius square and Mie potentials by applying the Supersymmetry approach. This potential is one of the established improved empirical potential functions which are practically applicable in different areas including thermodynamic properties calculations. ${ }^{1,10}$ In addition, the behavior of $\mathrm{H}_{2}, \mathrm{I}_{2}, \mathrm{CO}, \mathrm{NO}$, and $\mathrm{HCl}$ molecules are investigated subject to the MMSP. ${ }^{2}$ The solutions of the Schrödinger equation for the modified Mobius square plus Kratzer potential have been obtained using the Nikiforov-Uvarov method and employing the approximation scheme for the centrifugal term. ${ }^{11}$ In 2013 , the solution of the Dirac equation with relativistic spin and pseudospin symmetries of inversely quadratic Yukawa-like plus Mobius square potentials including a Coulomb-like tensor interaction has been obtained using the Nikiforov-Uvarov (NU) method. ${ }^{12}$ The Mobius square potential is considered a candidate par excellence to study the influences between diatomic molecules. ${ }^{13}$ Relativistic and relativistic quantum mechanics has achieved great successes in terms of the convergence of theoretical treatments with experimental measurements. However, until now, some data and indications remain that point strongly to unresolved cases based on quantum mechanics for example the non-renormalizable of electroweak interactions, the non-regularization of quantum field theories, quantum gravity, string theory. The idea of non-commutativity resulting from properties of deformation of space-space (Heisenberg in 1930 is the first to suggest the idea and then it was developed by Snyder in 1947) was one of the major solutions to these problems, As a result of all these motivational data, it is logical to consider the topographical properties of the noncommutativity space-space and phase-phase have a clear effect on the various physical properties of relativistic and nonrelativis- tic quantum systems. ${ }^{14-29}$

The above works motivated us to investigate the approximate solutions of the 3-dimensional deformed Klein-Gordon equation (DKGE, in short), the deformed Dirac equation (DDE, in short) and deformed Schrödinger equation (DSE, in short) for the improved modified Mobius square potential model offered by Okorie et al., ${ }^{1}$ Onyenegecha et al., ${ }^{2}$ and Ikot et $a .^{3}$ in the relativistic regime and nonrelativistic regime in the context of ordinary quantum mechanics. The potential under study can be applied for some selected diatomic molecules such as $\left(\mathrm{H}_{2}, \mathrm{I}_{2}, \mathrm{CO}, \mathrm{NO}\right.$, and $\left.\mathrm{HCl}\right)$ in RNCQM and NRNCQM symmetries. We hope to discover more investigations on the microscopic scale and from achieving more scientific knowledge of elementary particles in the field of nano-scales. The relativistic and nonrelativistic energy levels under the modified Mobius squared potential model have not been obtained yet in the RNCQM and NRNCQM symmetries, we hope to find new applications and profound physical interpretations using a new version model of the improved modified Mobius squared potential, this potential modeled in the new symmetries of NCQM as follows:

$$
\left\{\begin{array}{l}
V_{m s p}\left(r_{n c}\right)=V_{m s p}(r)-\frac{\partial V_{m s p}(r)}{\partial r} \frac{\mathbf{L} \Theta}{2 r}+O\left(\Theta^{2}\right) \\
S_{m s p}\left(r_{n c}\right)=S_{m s p}(r)-\frac{\partial S_{m s p}(r)}{\partial r} \frac{\mathbf{L} \Theta}{2 r}+O\left(\Theta^{2}\right)
\end{array}\right.
$$

Where

$$
\begin{gathered}
V_{m s p}(r)=-V_{0}\left(\frac{A+B e^{-2 a r}}{1-e^{-2 \alpha r}}\right)^{2} \\
\text { and } \quad S_{m s p}(r)=-S_{0}\left(\frac{A+B e^{-2 a r}}{1-e^{-2 a r}}\right)^{2}
\end{gathered}
$$

where $A, B, \alpha,\left(V_{0}, S_{0}\right)$ are the potential depths, $\left(r_{n c}\right.$ and $r$ ) are the distances between the two particles in NCQM and $\mathrm{QM}$ symmetries. The coupling $\mathbf{L} \Theta \equiv \vec{L} \vec{\Theta}$ equals $L_{x} \Theta_{12}+L_{y} \Theta_{23}+L_{z} \Theta_{13}$ with $L_{x}, L_{y}$ and $L_{z}$ are present the usual components of the angular momentum operator $L$ while the new noncommutativity parameter $\Theta_{i j}$ equals $\theta_{i j} / 2$. The new algebraic structure of covariant noncommutative canonical commutations relations NCNCCRs in the three representations of Schrödinger, Heisenberg, and interaction picture in the new symmetry of NCQM, as follows ${ }^{30-36}$ :

$$
\begin{gathered}
{\left[x_{\mu}^{(S, H, I)}, p_{\nu}^{(S, H, I)}\right]=i \hbar \delta_{\mu v}} \\
\Rightarrow\left[x_{n c_{\mu}}^{(S, H, I)_{*}}, p_{n c_{\nu}}^{(S, H, I)}\right]=i \hbar \delta_{\mu v} \\
{\left[x_{\mu}^{(S, H, I)}, x_{\nu}^{(S, H, I)}\right]=0 \Rightarrow\left[x_{n c_{\mu}}^{(S, H, I)_{*}}, x_{n c_{\nu}}^{(S, H, I)}\right]=i \theta_{\mu \nu}}
\end{gathered}
$$

With $\quad x_{n c \mu}^{(S, H, I)}=\left(x_{n c \mu}^{S}, x_{n c \mu}^{H}, x_{n c \mu}^{I}\right) \quad$ and $\quad p_{n c \mu}^{(S, H, I)}$ $=\left(p_{n c \mu}^{S}, p_{n c \mu}^{H}, p_{n c \mu}^{I}\right)$. It is important to note that Eq. (4) is a covariant equation (the same behavior of $x^{\mu}$ ) under Lorentz transformation, which includes boosts and/or rotations of the observer's inertial frame. We generalize the NCNCCRs to include Heisenberg and interaction pictures. It should be noted that, in our calculation, we have used natural units $\hbar=c=1$. Here $\hbar_{e f f} \cong \hbar$ is the effective Planck constant, $\theta_{\mu v}=\varepsilon_{\mu v} \theta(\theta$ is the noncommutative parameter) which is an infinitesimal parameter if compared to the energy values and elements of antisymmetric $3 X 3$ real matrices and $\delta_{\mu v}$ is the identity matrix. The symbol $*$ denotes the Weyl Moyal star product, which is generalized between two ordinary functions $f(x) h(x)$ to the new deformed form $\hat{f}\left(x_{n c}\right) \hat{h}\left(x_{n c}\right)$ 
which expressed with the Weyl Moyal star product $f(x) * h(x)$ in the symmetries of NCQM as follows ${ }^{37-43}$ :

$$
\begin{aligned}
(f * h)(x) \approx & (f h)(x)-\left.\frac{i \varepsilon^{\mu v} \theta}{2} \partial_{\mu}^{x} f \partial_{\nu}^{x} h\right|_{x^{\mu}=x^{\nu}} \\
& +O\left(\theta^{2}\right)
\end{aligned}
$$

The indices $(\mu, \nu=1,2,3)$ and $O\left(\theta^{2}\right)$ stand for the second and higher-order terms of the NC parameter. Physically, the second term in Eq. (5) presents the effects of space-space noncommutativity. Furthermore, it is possible to unify the operators $\quad \Omega_{n c \mu}^{H}(t)=\left(x_{n c \mu}^{H} \vee p_{n c \mu}^{H}\right)(t) \quad$ and $\quad \Omega_{n c \mu}^{I}(t)$ $=\left(x_{n c \mu}^{I} \vee p_{n c \mu}^{I}\right)(t)$ in the Heisenberg and the interaction pictures using the following projection relations, respectively:

$$
\left\{\begin{array}{rc}
\Omega_{n c \mu}{ }_{n}(t)= & \exp \left(i H_{r n c}^{m s p} T\right) * \Omega_{n c}{ }^{S} \\
& * \exp \left(-i H_{r n c}^{m s p} T\right) \\
\Omega_{n c \mu}(t)= & \exp \left(i H_{o n c}^{m s p} T\right) * \Omega_{n c \mu}{ }_{n c} \\
& * \exp \left(-i H_{o n c}^{m s p} T\right)
\end{array}\right.
$$

Where the corresponding operators in usual quantum mechanics are given by:

$$
\left\{\begin{array}{c}
\Omega_{\mu}^{H}(t)=\exp \left(i H_{r}^{m s p} T\right) \zeta_{\mu}^{S} \exp \left(-i H_{r}^{m s p} T\right) \\
\Omega_{\mu}^{I}(t)=\exp \left(i H_{o}^{m s p} T\right) \Omega_{\mu}^{S}(t) \exp \left(-i H_{o}^{m s p} T\right)
\end{array}\right.
$$

Where $\Omega_{\mu}^{S}=x_{\mu}^{S} \vee p_{\mu}^{S}$ are the operators in the Schrödinger picture, $\Omega_{\mu}^{H}=\left(x_{\mu}^{H} \vee p_{\mu}^{H}\right)(t)$ and $\Omega_{\mu}^{I}=\left(x_{\mu}^{I} \vee p_{\mu}^{I}\right)(t)$ are the Heisenberg and the interaction pictures in the ordinary QM symmetries. Moreover, the dynamics of new systems $\frac{d \Omega_{n a t}^{H}(t)}{d t}$ can be described by the following motion equations in the deformed Heisenberg picture as follows:

$$
\begin{gathered}
\frac{d \Omega_{\mu}^{H}(t)}{d t}=-i\left[\Omega_{\mu}^{H}(t), H_{r m s p}\right]+\frac{\partial \Omega_{\mu}^{H}(t)}{\partial t} \\
\Rightarrow \frac{d \Omega_{n \mu}^{H}(t)}{d t}=-i\left[\Omega_{n c \mu}^{H}(t), H_{r n c}^{m s p}\right]++\frac{\partial \Omega_{n \mu}^{H}(t)}{\partial t}
\end{gathered}
$$

Here $\left(H_{o n c}^{m s p}\right.$ and $\left.H_{r n c}^{m s p}\right)$ are the free and total Hamiltonian operators for the modified Mobius squared potential while $\left(H_{o}^{m s p}\right.$ and $\left.H_{r}^{m s p}\right)$ the corresponding Hamiltonians in the symmetries of NCQM. The present investigation aims at constructing a relativistic noncommutative effective scheme for the modified Mobius square potential model. It should be noted that the new commutators of the generators $L_{x}, L_{y}$ and $L_{z}$ in the new symmetries modified to become as follows:

$$
\left[L_{\alpha}, L_{\beta}\right]=i \varepsilon_{\alpha \beta}^{\gamma} L_{\gamma} \Rightarrow\left[L_{\alpha}^{*}, L_{\beta}\right]=i \varepsilon_{\alpha \beta}^{\gamma} L_{\gamma}
$$

These generators form a three-dimensional modified Liealgebra of the extended group $S O$ (3). The new bilinear product $\left[L_{\alpha}^{*}, L_{\beta}\right]$ will satisfy the modified antisymmetries and Jacobi identity following properties:

$$
\left[L_{\alpha}, L_{\beta}\right]=-\left[L_{\beta}, L_{\alpha}\right] \Rightarrow\left[L_{\alpha}^{*}, L_{\beta}\right]=-\left[L_{\beta}^{*}, L_{\alpha}\right]
$$

and

$$
\begin{aligned}
{\left[L_{\gamma} *\left[L_{\alpha}, L_{\beta}\right]\right]+} & -\left[L_{\beta}{ }^{*}\left[L_{\gamma}{ }^{*}, L_{\alpha}\right]\right] \\
& +\left[L_{\alpha},\left[L_{\beta}, L_{\gamma}\right]\right]=0
\end{aligned}
$$

On the other hand, the choice of the modified Mobius square potential from the fact that it exhibits an almost exact behavior similar to the Morse potential ${ }^{44}$ and Deng-Fan potential ${ }^{45}$ and so considers it an excellent choice for the study of atomic interactions for diatomic molecules such as $\mathrm{H}_{2}, \mathrm{I}_{2}, \mathrm{CO}, \mathrm{NO}$, and $\mathrm{HCl}$. Our current work is structured in eight sections. The first one includes the scope and purpose of our investigation, while the remaining parts of the paper are structured as follows. A review of the relativistic Klien-
Gordon equation RKGE, relativistic Dirac equation RDE, and nonrelativistic Schrödinger equation NRSE with the modified Mobius square potential. Sect. 3 is devoted to studying the DRKGE by applying the ordinary Bopp's shift method and the Greene and Aldrich approximation for the centrifugal term to obtain the effective potential of the improved modified Mobius squared potential. Besides, via perturbation theory, we find the expectation values of some radial terms to calculate the energy shift produced by the effect of the perturbed effective potential on the modified Mobius squared potential. Sect. 4 is devoted to present the global energy shift and the global energy spectra produced by the modified Mobius squared potential in the RNCQM symmetries. In Sect. 5, we summarized the spectrum of relativistic deformed Klien-Gordon theory under the modified Mobius squared potential model. In Sect. 6, we will study the fermionic massive spin $1 / 2$ particles interacting with the relativistic improved modified Mobius square potential model in the deformed Dirac theory. In the next section, we will investigate the improved modified Mobius square potential model in the deformed Schrödinger theory and Sect. 8 gives the conclusion of the paper.

\section{REVISED OF RKGE, RDE, AND NRSE UNDER THE MODIFIED MOBIUS SQUARED POTENTIAL MODEL}

Before we start constructing the new solutions of the deformed Klein-Gordon theory, the deformed Dirac theory, and the deformed Schrödinger theory under the improved modified Mobius squared potential model, we give a summary of the corresponding usual solutions in ordinary relativistic quantum mechanics RQM and nonrelativistic quantum mechanics NRQM. Given modified Mobius squared potential model in the symmetries of RQM and NRQM by the following versions ${ }^{1-3}$ :

$$
\begin{aligned}
V_{m s p}(r) & =-V_{0}\left(\frac{A+B e^{-2 \alpha r}}{1-e^{-2 \alpha r}}\right)^{2} \\
& =-V_{0}\left(\frac{A^{2}+2 A B e^{-2 \alpha r}+B^{2} e^{-4 \alpha r}}{\left(1-e^{-2 \alpha r}\right)^{2}}\right)
\end{aligned}
$$

and

$$
\begin{aligned}
S_{m s p}(r) & =-S_{0}\left(\frac{A+B e^{-2 \alpha r}}{1-e^{-2 \alpha r}}\right)^{2} \\
& =-S_{0}\left(\frac{A^{2}+2 A B e^{-2 \alpha r}+B^{2} e^{-4 \alpha r}}{\left(1-e^{-2 \alpha r}\right)^{2}}\right)
\end{aligned}
$$

The Mobius square potential is the more general case of both Hulthén and Morse potentials. ${ }^{46,47}$ The 3-dimensional Klein-Gordon, Dirac equations with $S_{m s p}(r)$ and a vector potential $V_{m s p}(r)$ and the Schrödinger equation with the vector potential $V_{m s p}(r)$ for the diatomic molecule $\mathrm{H}_{2}, \mathrm{I}_{2}$, $\mathrm{CO}, \mathrm{NO}$ and $\mathrm{HCl}$ with reduced mass $M$ (or fermionic particles) are given as:

$$
\begin{aligned}
(-\Delta & +\left(M+S_{m s p}(r)\right)^{2} \\
& \left.-\left(E_{n l}^{k g}-V_{m s p}(r)\right)^{2}\right) \Psi(r, \theta, \phi)=0
\end{aligned}
$$




$$
\begin{gathered}
\left(\alpha p+\beta\left(M+S_{m s p}(r)\right)\right) \Psi_{n k}(r, \theta, \phi) \\
=\left(E_{n k}-V_{m s p}(r)\right) \Psi_{n k}(r, \theta, \phi) \\
\left(-\frac{\Delta}{2 M}+V_{m s p}(r)\right) \Psi(r, \theta, \phi)=E_{n l}^{n r} \Psi(r, \theta, \phi)
\end{gathered}
$$

Here $\Delta$ is the ordinary 3-dimensional Laplacian operator, the vector potential $V_{m s p}(r)$ due to the four-vector linear momentum operator $A^{\mu}\left(V_{m s p}(r), A=0\right)$ and space-time scalar potential $S_{m s p}(r)$ due to the mass, $\left(E_{n l}, E_{n k}\right)$ and $E_{n l}^{n r}$ are represents the relativistic and nonrelativistic energy eigenvalues, $(n, l)$ are represents the principal and orbital quantum numbers, respectively, $\alpha_{i}=\left(\begin{array}{cc}0 & \sigma_{i} \\ \sigma_{i} & 0\end{array}\right)$, $\beta=\left(\begin{array}{cc}I_{2 \times 2} & 0 \\ 0 & I_{2 \times 2}\end{array}\right)$ and $\sigma_{i}$ are the usual Dirac matrices. Since the modified Mobius squared potential has spherical symmetry, allowing the solutions of the time-independent Klein-Gordon equation and Schrödinger equation of the known form $\Psi(r, \theta, \phi)=\frac{U_{n l}(r)}{r} Y_{l}^{m}(\theta, \phi)$ to separate the radial $U_{n l}(r)$ and angular parts $Y_{l}^{m}(\theta, \phi)$ of the wave function $\Psi(r, \theta, \phi)$. For the Dirac equation $\Psi_{n k}(r, \theta, \phi)$ $=\frac{1}{r}\left(\begin{array}{c}F_{n k}(r) Y_{j m}^{l}(\theta, \phi) \\ G_{n k}(r) Y_{j m}^{\tilde{l}}(\theta, \phi)\end{array}\right)$, where $F_{n k}(r)$ and $G_{n k}(r)$ represent the upper and lower components of the Dirac spinors $\Psi_{n k}(r, \theta, \phi)$ while $Y_{j m}^{l}(\theta, \phi)$ and $Y_{j m}^{\tilde{l}}(\theta, \phi)$ are the spin and pseudospin spherical harmonics and $m$ is the projection on the $O Z$ axis. Thus, the radial part of Eqs. (13), (14), and (15) can be expressed as:

$$
\begin{aligned}
& \left(\frac{d^{2}}{d r^{2}}-\left(M^{2}-E_{n l}^{2}\right)-2\left(E_{n l} V_{m s p}(r)\right.\right. \\
& \left.+M S_{m s p}(r)\right)+V_{m s p}^{2}(r) \\
& \left.-S_{m s p}^{2}(r)-\frac{l(l+1)}{r^{2}}\right) U_{n l}(r)=0 \\
& {\left[\frac{d^{2}}{d r^{2}}-\frac{k(k+1)}{r^{2}}+\frac{2 k U(r)}{r}\right.} \\
& -\frac{d U(r)}{d r}-U^{2}(r) \\
& -\left(M+E_{n k}-\Delta(r)\right) \\
& \cdot\left(M-E_{n k}+\Sigma(r)\right) \\
& \left.+\frac{\frac{d \Delta(r)}{d r}\left(\frac{d}{d r}+\frac{k}{r}-U(r)\right)}{\left(M+E_{n k}-\Delta(r)\right)}\right] F_{n k}(r)=0 \\
& {\left[\frac{d^{2}}{d r^{2}}-\frac{k(k-1)}{r^{2}}+\frac{2 k U(r)}{r}+\frac{d U(r)}{d r}\right.} \\
& -U^{2}(r)-\left(M+E_{n k}-\Delta(r)\right) \\
& \text { - }\left(M-E_{n k}+\Sigma(r)\right) \\
& \left.+\frac{\frac{d \Sigma(r)}{d r}\left(\frac{d}{d r}-\frac{k}{r}+U(r)\right)}{\left(M+E_{n k}-\Sigma(r)\right)}\right] G_{n k}(r)=0 \\
& \left(\frac{d^{2}}{d r^{2}}+2 M\left(E_{n l}^{n r}-V_{m s p}(r)\right.\right. \\
& \left.\left.-\frac{l(l+1)}{r^{2}}\right)\right) U_{n l}(r)=0
\end{aligned}
$$

With $\quad k(k-1)=\tilde{l}(\tilde{l}-1) \quad$ and $\quad k(k+1)=l(l+1)$, $U(r)=-V_{1}\left(\frac{e^{-a r}}{r}\right)$ is the Yukawa tensor interaction, in meson physics, $V_{1}$ and $\alpha$ are strength and range of the nucleon force, respectively. Using the shorthand notation

$$
\begin{gathered}
E_{e f f}^{m s p}=M^{2}-E_{n l}^{2}, \text { and } \\
V_{e f f}^{m s p}(r)=2\left(E_{n l} V_{m s p}(r)+M S_{m s p}(r)\right) \\
-V_{m s p}^{2}(r)+S_{m s p}^{2}(r)+\frac{l(l+1)}{r^{2}} \\
V_{e f f-n r}^{m s p}(r)=V_{m s p}(r)-\frac{l(l+1)}{r^{2}} \\
\begin{array}{c}
\Sigma(r)=-V_{0}\left(\frac{A+B e^{-2 a r}}{1-e^{-2 a r}}\right)^{2} \text { and } \\
\frac{d \Delta(r)}{d r}=0 \Rightarrow \Delta(r)=C_{s}
\end{array}
\end{gathered}
$$

For Spin Symmetry Limit

$$
\begin{gathered}
\Delta(r)=-V_{0}\left(\frac{A+B e^{-2 \alpha r}}{1-e^{-2 \alpha r}}\right)^{2} \text { and } \\
\frac{d \Sigma(r)}{d r}=0 \Rightarrow \Sigma(r)=C_{p s}
\end{gathered}
$$

For Pseudospin Symmetry Limit

The authors Onyenegecha et al. ${ }^{2}$ have adopted Alhaidari et al.'s scheme and rescale the potential under the non-relativistic limit as $V_{m p}(r)=S_{m p}(r) \mapsto \frac{V_{m p}(r)}{2}$, the effective potential of the modified Mobius squared potential model reduces to the form:

$$
\begin{aligned}
V_{e f f}^{m s p}(r)= & -V_{0}\left(E_{n l}+M\right)\left(\frac{A+B e^{-2 \alpha r}}{1-e^{-2 \alpha r}}\right)^{2} \\
& +\frac{l(l+1)}{r^{2}} \\
V_{e f f-n r}^{m s p}(r)= & -V_{0}\left(\frac{A+B e^{-2 \alpha r}}{1-e^{-2 \alpha r}}\right)^{2}-\frac{l(l+1)}{r^{2}}
\end{aligned}
$$

We obtain the following second-order Schrödinger-like equation:

$$
\begin{aligned}
& \left(\frac{d^{2}}{d r^{2}}-\left(E_{e f f}^{m s p}+V_{e f f}^{m s p}(r)\right)\right) U_{n l}(r)=0 \\
& {\left[\begin{array}{c}
\frac{d^{2}}{d r^{2}}-\frac{k(k+1)}{r^{2}}+\frac{2 k U(r)}{r} \\
-\frac{d U(r)}{d r}-U^{2}(r) \\
-\left(M+E_{n k}-C_{s}\right) \\
\cdot\left(M-E_{n k}-V_{0}\left(\frac{A+B e^{-2 a r}}{1-e^{-2 \alpha r}}\right)^{2}\right)
\end{array}\right] F_{n k}(r)=0} \\
& {\left[\begin{array}{c}
\frac{d^{2}}{d r^{2}}-\frac{k(k-1)}{r^{2}}+\frac{2 k U(r)}{r} \\
+\frac{d U(r)}{d r}-U^{2}(r) \\
-\left(M+E_{n k}+V_{0}\left(\frac{A+B e^{-2 a r}}{1-e^{-2 \alpha r}}\right)^{2}\right) \\
\cdot\left(M-E_{n k}+C_{p s}\right)
\end{array}\right] G_{n k}(r)=0} \\
& \left(\frac{d^{2}}{d r^{2}}+2 M\left(E_{n l}^{n r}-V_{e f f-n r}^{m s p}(r)\right)\right) U_{n l}(r)=0
\end{aligned}
$$

Okorie et al., ${ }^{1}$ Onyenegecha et al., ${ }^{2}$ and Ikot et al. ${ }^{3}$ using both Nikiforov-Uvarov method and the Greene and Aldrich approximation for the centrifugal term ${ }^{48}$ to obtain the expressions for the wave function as hypergeometric polynomials $2 F_{1}\left(-n, n+a_{n}+b_{n}+1 ; 1+a_{n}, s\right)$ and the corresponding energy values for the modified Mobius squared potential, in RQM and NRQM symmetries as,

$$
\begin{aligned}
\Psi(r, \theta, \phi)= & \frac{N_{n l}}{r} s^{\sqrt{\Lambda_{n l}}}(1-s)^{\chi_{n l}} P_{n}^{\left(2 \sqrt{\Lambda_{n l}}, 2 \chi_{n l}-1\right)} \\
& \cdot(1-2 s) Y_{l}^{m}(\theta, \phi)
\end{aligned}
$$




$$
\begin{aligned}
F_{n k}(r)= & N_{n k} s^{\sqrt{D_{3}^{s}}}(1-s)^{1 / 2+\sqrt{1 / 4+D_{1}^{s}+D_{3}^{s}-D_{2}^{s}}} \\
& \cdot 2 F_{1}\left(-n, n+2 \sqrt{D_{3}^{s}}\right. \\
& +2 \sqrt{1 / 4+D_{1}^{s}+D_{3}^{s}-D_{2}^{s}} \\
& \left.+1 ; 1+2 \sqrt{D_{3}^{s}} ; s\right) \\
G_{n k}(r)= & N_{n k} s^{\sqrt{Q_{3}^{p s}}}(1-s)_{2}^{1 / 2+\sqrt{1 / 4+Q_{1}^{p s}+Q_{3}^{p s}-Q_{2}^{p s}}} \\
& \cdot F_{1}\left(-n, n+2 \sqrt{Q_{3}^{p s}}\right. \\
& +2 \sqrt{1 / 4+Q_{1}^{p s}+Q_{3}^{p s}-Q_{2}^{p s}} \\
& \left.+1 ; 1+2 \sqrt{Q_{3}^{p s}} ; s\right)
\end{aligned}
$$

Where $\quad \Lambda_{n l}=\frac{\left(E_{n l}^{2}-M^{2}\right)+V_{0} B^{2}\left(E_{n l}+M\right)}{4 \alpha^{2}}, \quad \chi_{n l}=\frac{1}{2}$ $+\frac{1}{2} \sqrt{(2 l+1)^{2}-\frac{V_{0}(A+B)^{2}\left(E_{n l}+M\right)}{\alpha^{2}}}, \quad D_{1}^{s}=V_{1}\left(V_{1}-1 / 2\right)-\frac{\delta^{2}}{4 \alpha^{2}}$ $-\frac{\gamma^{2} B^{2}}{4 \alpha^{2}}, D_{2}^{s}=-\left(\frac{2 \delta^{2}}{4 \alpha^{2}}+\frac{2 A B \gamma^{2}}{4 \alpha^{2}}+k(k+1)+(2 k+3 / 2) V_{1}\right), D_{3}^{s}$ $=-\left(\frac{\delta^{2}}{4 \alpha^{2}}-\frac{A^{2} \gamma^{2}}{4 \alpha^{2}}\right), \delta^{2}=\left(-M-E_{n k}^{s}+C_{s}\right)\left(-M-E_{n k}^{s}\right), \gamma^{2}$ $=\left(-M-E_{n k}^{s}+C_{s}\right) V_{0}, \quad Q_{1}^{p s}=V_{1}\left(V_{1}+1 / 2\right) \quad-\frac{\varepsilon^{2}}{4 \alpha^{2}}-\frac{\beta^{2} B^{2}}{4 \alpha^{2}}$, $Q_{2}^{p s}=-\left(\frac{2 \varepsilon^{2}}{4 \alpha^{2}}-\frac{2 A B \beta^{2}}{4 \alpha^{2}} \quad+k(k-1)+(2 k-3 / 2) V_{1}\right), \quad Q_{3}^{p s}$ $=-\left(\frac{\varepsilon^{2}}{4 \alpha^{2}}-\frac{A^{2} \beta^{2}}{4 \alpha^{2}}\right), \quad \varepsilon^{2}=\left(-M+E_{n k}^{p s}-C_{p s}\right)\left(M+E_{n k}^{p s}\right)$, $s=e^{-2 \alpha r},{ }_{2} F_{1}(X)$ are the hypergeometric polynomials while $N_{n l}$ and $N_{n k}$ are the normalization constants and the energy eigenvalues are given by:

$$
\begin{aligned}
E_{n l}^{2} & -M^{2} \\
= & -V_{0} A^{2}\left(E_{n l}+M\right) \\
& -4 \alpha^{2}\left\{\left[\frac{-V_{0} A(A B)\left(E_{n l}+M\right)}{2 \alpha^{2}}\right.\right. \\
& +\frac{1}{2}+n(n+1) \\
& +\left(n+\frac{1}{2}\right) \\
& +\sqrt{\left.(2 l+1)^{2}-\frac{V_{0}(A+B)^{2}\left(E_{n l}+M\right)}{2 \alpha^{2}}\right]} \\
& \div[1+2 n \\
& \left.+\sqrt{\left.(2 l+1)^{2}-\frac{V_{0}(A+B)^{2}\left(E_{n l}+M\right)}{2 \alpha^{2}}\right]}\right\}
\end{aligned}
$$

The energy eigenvalues for spin symmetry limit as:

$$
\begin{aligned}
n & +\frac{2 n+1}{2}+(2 n+1)\left(\sqrt{1 / 4+D_{1}^{s}+D_{3}^{s}-D_{2}^{s}}\right. \\
& \left.+\sqrt{D_{3}^{s}}\right)+n(n-1) \\
= & D_{2}^{s}-2 D_{3}^{s}-2 \sqrt{D_{3}^{s}\left(1 / 4+D_{1}^{s}+D_{3}^{s}-D_{2}^{s}\right)}
\end{aligned}
$$

The energy eigenvalues for spin pseudospin symmetry limit as:

$$
\begin{aligned}
n+ & \frac{2 n+1}{2}+(2 n+1) \\
& \cdot\left(\sqrt{1 / 4+Q_{1}^{p s}+Q_{3}^{p s}-Q_{2}^{p s}}+\sqrt{Q_{3}^{p s}}\right) \\
& +n(n-1) \\
= & Q_{2}^{p s}-2 Q_{3}^{p s} \\
& -2 \sqrt{Q_{3}^{p s}\left(1 / 4+Q_{1}^{p s}+Q_{3}^{p s}-Q_{2}^{p s}\right)}
\end{aligned}
$$

The nonrelativistic energy eigenvalues as ${ }^{1}$ :

$$
\begin{aligned}
E_{n l}^{n r}= & -\frac{\alpha^{2}}{2 M}\left(\left[\frac{1}{4}-\frac{M A B V_{0}}{\alpha^{2}}\right.\right. \\
& \left.-\frac{M V_{0} A^{2}}{2 \alpha^{2}} \frac{M V_{0} B^{2}}{2 \alpha^{2}}+l(l+1)\right]^{1 / 2} \\
& -(n+1 / 2)+\left[\frac { M } { 2 \alpha ^ { 2 } } \left(V_{0} B^{2}\right.\right. \\
& \left.\left.-V_{0} A^{2}+\frac{2 \alpha^{2} l(l+1)}{M}\right)\right] \\
& \div\left\{\left[\frac{1}{4}-\frac{M A B V_{0}}{\alpha^{2}}\right.\right. \\
& \left.-\frac{M V_{0} A^{2}}{2 \alpha^{2}} \frac{M V_{0} B^{2}}{2 \alpha^{2}}+l(l+1)\right]^{1 / 2} \\
& -(n+1 / 2)\})^{2}-V_{0} A^{2}+\frac{2 \alpha^{2} l(l+1)}{M}
\end{aligned}
$$

From the definition of Jacobi polynomials ${ }^{49}$ :

$$
\begin{aligned}
P_{n}^{\left(a_{n}, b_{n}\right)}(1-2 s)= & \frac{\Gamma\left(n+a_{n}+1\right)}{n ! \Gamma\left(a_{n}+1\right)} \\
& \cdot{ }_{2} F_{1}\left(-n, n+a_{n}\right. \\
& \left.+b_{n}+1 ; 1+a_{n}, s\right)
\end{aligned}
$$

In terms of the definition of the hypergeometric polynomials, Eq. (25) can be written as:

$$
\begin{aligned}
\Psi(r, \theta, \phi)= & \frac{\Gamma\left(n+2 \sqrt{\Lambda_{n l}}+1\right)}{n ! \Gamma\left(2 \sqrt{\Lambda_{n l}}+1\right)} \frac{N_{n l}}{r} s^{\sqrt{\Lambda_{n l}}} \\
& \cdot(1-s)^{\chi_{n l}} F_{1}\left(-n, n+2 \sqrt{\Lambda_{n l}}\right. \\
& \left.+2 \chi_{n l} ; 1+2 \sqrt{\Lambda_{n l}}, s\right) Y_{l}^{m}(\theta, \phi)
\end{aligned}
$$

\section{THE SOLUTION OF DRKGE UNDER THE IMSPM IN DRKGT SYMMETRIES}

\subsection{REVIEW OF BOPP'S SHIFT METHOD}

At the beginning of this subsection, we shall give and define a formula of the improved modified Mobius squared potential model in the symmetries of deformed relativistic KlienGordon theory DRKGT symmetries. To achieve this goal, it is useful to write the deformed relativistic Klien-Gordon equation DKGE by applying the notion of the Weyl Moyal star product which has been seen previously in Eqs. (3-10) with the differential equation that is satisfied by the radial wave function $U_{n l}(r)$ in Eq. (16), Thus, the radial wave function in DRKGTS symmetries becomes as follows ${ }^{50-56}$ :

$$
\left(\frac{d^{2}}{d r^{2}}-\left(E_{e f f}^{m s p}+V_{e f f}^{m s p}(r)\right)\right) * U_{n l}(r)=0
$$

It is established extensively in the literature and a basic text that star products can be simplified by Bopp's shift method. 17,33,57-59 The physicist Fritz Bopp was the first to consider pseudo-differential operators obtained from a symbol by the quantization rules $x \rightarrow x-\frac{i}{2} \frac{\partial}{\partial p}$ and $p \rightarrow p+\frac{i}{2} \frac{\partial}{\partial x}$ instead of the ordinary correspondence $x \rightarrow x$ and $p \rightarrow \frac{i}{2} \frac{\partial}{\partial x} \cdot 58,59$ In physics literature, this is known by Bopp's shifts. This quantization procedure is called Bopp quantization. It is known to specialists that Bopp's shift method, has been applied effectively and has succeeded in simplifying the three basic equations: DSE, ${ }^{60-64}$ DKGE, ${ }^{50-56,65,66}$ and deformed Dirac equation $\mathrm{DDE}^{67-70}$ with the notion of star product to the Schrödinger 
equation SE, KGE and Dirac equation DE with the notion of ordinary product, respectively. Thus, Bopp's shift method is based on reducing second-order linear differential equations of DSE, DKGE, and DDE with star product to secondorder linear differential equations of SE, KGE, and DE without star products with simultaneous translation in the space-space. The CNCCRs with star product in Eqs. (3) and (4) become new CNCCRs without the notion of star product as follows (see, e.g., 57-60,65,66,71):

$$
\begin{aligned}
& {\left[x_{n c \mu}^{(S, H, I)}, p_{n c \nu}{ }^{(S, H, I)}\right]=i \hbar_{e f f} \delta_{\mu \nu}} \\
& \text { and }\left[x_{n c \mu}{ }^{(S, H, I)}, x_{n c \nu}{ }^{(S, H, I)}\right]=i \theta_{\mu \nu}
\end{aligned}
$$

The generalized positions and momentum coordinates $x_{n c \mu}^{(S, H, I)}=\left(x_{n c \mu}^{S}, x_{n c \mu}^{H}, x_{n c \mu}^{I}\right)$ and $p_{n c \mu}^{(S, H, I)}=\left(p_{n c \mu}^{S}, p_{n c \mu}^{H}, p_{n c \mu}^{I}\right)$, in the symmetries of DRKGT are defined in terms of the corresponding coordinates in the symmetries of RQM $x_{\mu}^{(S, H, I)}=\left(x_{\mu}^{S}, x_{\mu}^{H}, x_{\mu}^{I}\right)$ and $p_{\mu}^{(S, H, I)}=\left(p_{\mu}^{S}, p_{\mu}^{H}, p_{\mu}^{I}\right)$ via, respectively 60,65 :

$$
\begin{gathered}
x_{n c \mu}{ }^{(S, H, I)}=x_{\mu}^{(S, H, I)}-\sum_{\nu=1}^{3} \frac{i \theta_{\mu v}}{2} p_{\nu}^{(S, H, I)} \\
\text { and } p_{n c \mu}{ }^{(S, H, I)}=p_{\mu}^{(S, H, I)}
\end{gathered}
$$

This allows us to find the operator $r_{n c}^{2}$ equal $r^{2}-L \Theta$ in NCQM symmetries. ${ }^{50-54}$

\subsection{THE NEW EFFECTIVE POTENTIAL OF THE IMSPM IN DRKGT SYMMETRIES}

According to the Bopp shift method, Eq. (45) becomes similar to the following like the Schrödinger equation (without the notions of star product):

$$
\left(\begin{array}{c}
\frac{d^{2}}{d r^{2}}--\left(M^{2}-E_{n l}^{2}\right)-\frac{l(l+1)}{r_{n c}^{2}} \\
-V_{m s p}\left(r_{n c}\right)\left(E_{n l}+M\right)
\end{array}\right) U_{n l}(r)=0
$$

The new operators $V_{m s p}\left(r_{n c}\right)$ and $\frac{l(l+1)}{r_{n c}^{2}}$ are expressed as in DRKGT symmetries as follows:

$$
\begin{gathered}
V_{m s p}\left(r_{n c}\right)=-V_{0}\left(\frac{A+B e^{-2 \alpha r}}{1-e^{-2 \alpha r}}\right)^{2} \\
-\frac{\partial V_{m s p}(r)}{\partial r} \frac{L \Theta}{2 r}+O\left(\Theta^{2}\right) \\
\frac{l(l+1)}{r_{n c}^{2}}=\frac{l(l+1)}{r^{2}}+\frac{l(l+1)}{r^{4}} L \Theta+O\left(\Theta^{2}\right)
\end{gathered}
$$

Therefore, we can rewrite:

$$
\begin{aligned}
V_{m s p}\left(r_{n c}\right)\left(E_{n l}+M\right)= & V_{m s p}(r)\left(E_{n l}+M\right) \\
& -\left(\frac{E_{n l}+M}{2 r}\right) \frac{\partial V_{m s p}(r)}{\partial r} \\
& \cdot L \Theta++O\left(\Theta^{2}\right)
\end{aligned}
$$

Moreover, to illustrate the above equation in a simple mathematical way and attractive form, it is useful to enter the following symbol $V_{n c-e f f}^{m s p}(r)$, thus the radial Eq. (37) becomes:

$$
\left(\frac{d^{2}}{d r^{2}}-\left(E_{e f f}^{m s p}+V_{n c-e f f}^{m s p}(r)\right)\right) U_{n l}(r)=0
$$

with $V_{n c-e f f}^{m s p}(r)=V_{e f f}^{m s p}(r)+V_{p e r t}^{m s p}(r)$. Moreover, $V_{p e r t}^{m s p}(r)$ is given by the following relation:

$$
\begin{aligned}
V_{p e r t}^{m s p}(r)= & \left(\frac{l(l+1)}{r^{4}}-\frac{E_{n l}+M}{2 r} \frac{\partial V_{m s p}(r)}{\partial r}\right) \\
& \cdot \mathbf{L} \Theta+O\left(\Theta^{2}\right)
\end{aligned}
$$

After straightforward calculations $\frac{\partial V_{m s p}(r)}{\partial r}$ we obtain as fol- lows:

$$
\begin{aligned}
\frac{\partial V_{m s p}(r)}{\partial r}= & \frac{4 V_{0} \alpha B A e^{-2 \alpha r}}{\left(1-e^{-2 \alpha r}\right)^{2}}+\frac{4 V_{0} \alpha B^{2} e^{-4 \alpha r}}{\left(1-e^{-2 \alpha r}\right)^{2}} \\
& -\frac{4 V_{0} \alpha A^{2} e^{-2 \alpha r}}{\left(1-e^{-2 \alpha r}\right)^{3}}-\frac{4 V_{0} \alpha B^{2} e^{-6 \alpha r}}{\left(1-e^{-2 \alpha r}\right)^{3}} \\
& \cdot \frac{-8 V_{0} A B \alpha e^{-4 \alpha r}}{\left(1-e^{-2 \alpha r}\right)^{3}}
\end{aligned}
$$

We insert Eqs. (43) into Eq. (42), this allows it to be rewritten $V_{\text {pert }}^{m s p}(r)$ in the following form:

$$
\begin{aligned}
V_{p e r t}^{m s p}(r)= & \left(\frac{l(l+1)}{r^{4}}-\left(\frac{E_{n l}+M}{2 r}\right) \alpha\right. \\
& \cdot\left(\frac{4 V_{0} A B e^{-2 \alpha r}}{\left(1-e^{-2 \alpha r}\right)^{2}}+\frac{4 V_{0} B^{2} e^{-4 \alpha r}}{\left(1-e^{-2 \alpha r}\right)^{2}}\right. \\
& -\frac{4 V_{0} A^{2} e^{-2 \alpha r}}{\left(1-e^{-2 \alpha r}\right)^{3}}-\frac{4 V_{0} B^{2} e^{-6 \alpha r}}{\left(1-e^{-2 \alpha r}\right)^{3}} \\
& \left.\left.-\frac{8 V_{0} A B e^{-4 \alpha r}}{\left(1-e^{-2 \alpha r}\right)^{3}}\right)\right) L \Theta+O\left(\Theta^{2}\right)
\end{aligned}
$$

It should be noted that Eq. (16) with the modified Mobius squared potential can be exactly solved for the s-wave $l=0$, but for the case $l \neq 0$, Onyenegecha et al. obtained approximate analytical solutions of the RKGE with the arbitrary $l \neq 0$ state using the Nikiforov-Uvarov method and employing the approximation scheme for the centrifugal term. ${ }^{2}$ In the new form of radial like-Schrödinger equation written in Eq. (41), we have new terms including $\frac{1}{r}, \frac{1}{r^{4}}$ and other likeCoulombic terms which make this equation impossible to solve analytically for $l=0$ and $l \neq 0$, it can only be solved approximately. From this point of view, we can consider the improved approximation of the centrifugal term proposed by M. Badawi et al., ${ }^{72}$ this method proved its power and efficiency when compared with Greene and Aldrich approximation. ${ }^{48}$ The approximations type suggested by (Greene and Aldrich) for a short-range potential is an excellent approximation to the centrifugal term and allows us to get a second-order solvable differential equation. Unlike the following approximation used in the previous works $1-3,50-56,65,66$ :

$$
\begin{aligned}
& \frac{1}{r^{2}} \approx \frac{4 \alpha^{2} e^{-2 \alpha r}}{\left(1-e^{-2 \alpha r}\right)^{2}}=\frac{4 \alpha^{2} s}{(1-s)^{2}} \\
& \Rightarrow \frac{1}{r} \approx \frac{2 \alpha e^{-\alpha r}}{1-e^{-2 \alpha r}}=\frac{2 \alpha s^{1 / 2}}{1-s}
\end{aligned}
$$

We point out here that the above approximation is only valid for small values of the screening parameter. By considering the transformation of the form $s=e^{-2 \alpha r}$, Eq. (44) now becomes:

$$
\begin{aligned}
V_{p e r t}^{m s p}(s)= & {\left[\frac{16 l(l+1) \alpha^{4} s^{2}}{(1-s)^{4}}-4\left(E_{n l}+M\right)\right.} \\
& \cdot \alpha^{2}\left(\frac{V_{0} A B s^{3 / 2}}{(1-s)^{3}}+\frac{V_{0} B^{2} s^{5 / 2}}{(1-s)^{3}}\right. \\
& -\frac{V_{0} A^{2} s^{3 / 2}}{(1-s)^{4}}-\frac{V_{0} B^{2} s^{7 / 2}}{(1-s)^{4}} \\
& \left.\left.-2 \frac{V_{0} A B s^{5 / 2}}{(1-s)^{4}}\right)\right] \mathbf{L \Theta}+O\left(\Theta^{2}\right)
\end{aligned}
$$

We have applied the approximations of Greene and Aldrich to the term $\frac{l(l+1)}{r^{4}}$. The modified Mobius squared potential model is extended by including new terms proportional to 
the radial terms $\frac{s^{2}}{(1-s)^{4}}, \frac{s^{3 / 2}}{(1-s)^{3}}, \frac{s^{5 / 2}}{(1-s)^{3}}, \frac{s^{3 / 2}}{(1-s)^{4}}, \frac{s^{7 / 2}}{(1-s)^{4}}$ and $\frac{s^{5 / 2}}{(1-s)^{4}}$ to become the improved modified Mobius squared potential model in RNCQM symmetries. The produced new effective potential $V_{n c-e f f}^{m s p}(s)$ is also proportional to the infinitesimal vector $\boldsymbol{\Theta}$. This allows us to consider the additive part $V_{p e r t}^{m s p}(s)$ as a perturbation potential compared with the main potential $V_{e f f}^{m s p}(s)$ (parent potential operator in the symmetries of RNCQM, that is, the inequality $V_{p e r t}^{m s p}(s)<<V_{e f f}^{m s p}(s)$ has become achieved. That is all physical justifications for applying the time-independent perturbation theory become satisfied. This allows us to give a complete prescription for determining the energy level of the generalized excited states.

\subsection{THE EXPECTATION VALUES OF IMSPM IN DRKGT SYMMETRIES}

In this subsection, we want to apply the perturbative theory, in the case of DRKGT symmetries, we find the expectation values of the radial terms $\frac{s^{2}}{(1-s)^{4}}, \frac{s^{3 / 2}}{(1-s)^{3}}, \frac{s^{5 / 2}}{(1-s)^{3}}, \frac{s^{3 / 2}}{(1-s)^{4}}$, $\frac{s^{7 / 2}}{(1-s)^{4}}$ and $\frac{s^{5 / 2}}{(1-s)^{4}}$ taking into account the wave function which we have seen previously in Eq. (33). Thus, after straightforward calculations, we obtain the following results:

$$
\begin{aligned}
& \left\langle\frac{s^{2}}{(1-s)^{4}}\right\rangle_{(n l m)}=N_{n l}^{\prime 2} \int_{0}^{+\infty}\left\{\frac{s^{2}}{(1-s)^{4}} s^{2 \sqrt{\Lambda_{n l}}}\right. \\
& \cdot(1-s)^{2 \chi_{n l}}\left[2 F_{1}(-n, n\right. \\
& +2 \sqrt{\Lambda_{n l}}+2 \chi_{n l} ; 1 \\
& \left.\left.\left.+2 \sqrt{\Lambda_{n l}}, s\right)\right]^{2}\right\} d r \\
& \left\langle\frac{s^{3 / 2}}{(1-s)^{3}}\right\rangle_{(n l m)}=N_{n l}^{\prime 2} \int_{0}^{+\infty}\left\{\frac{s^{3 / 2}}{(1-s)^{3}} s^{2 \sqrt{\Lambda_{n l}}}\right. \\
& \cdot(1-s)^{2 \chi_{n l}}\left[2 F_{1}(-n, n\right. \\
& +2 \sqrt{\Lambda_{n l}}+2 \chi_{n l} ; 1 \\
& \left.\left.\left.+2 \sqrt{\Lambda_{n l}}, s\right)\right]^{2}\right\} d r \\
& \left\langle\frac{s^{5 / 2}}{(1-s)^{3}}\right\rangle_{(n l m)}=N_{n l}^{\prime 2} \int_{0}^{+\infty}\left\{\frac{s^{5 / 2}}{(1-s)^{3}} s^{2 \sqrt{\Lambda_{n l}}}\right. \\
& \cdot(1-s)^{2 \chi_{n l}}\left[2 F_{1}(-n, n\right. \\
& +2 \sqrt{\Lambda_{n l}}+2 \chi_{n l} ; 1 \\
& \left.\left.\left.+2 \sqrt{\Lambda_{n l}}, s\right)\right]^{2}\right\} d r \\
& \left\langle\frac{s^{3 / 2}}{(1-s)^{4}}\right\rangle_{(n l m)}=N_{n l}^{\prime 2} \int_{0}^{+\infty}\left\{\frac{s^{3 / 2}}{(1-s)^{4}} s^{2 \sqrt{\Lambda_{n l}}}\right. \\
& \cdot(1-s)^{2 \chi_{n l}}\left[2 F_{1}(-n, n\right. \\
& +2 \sqrt{\Lambda_{n l}}+2 \chi_{n l} ; 1 \\
& \left.\left.\left.+2 \sqrt{\Lambda_{n l}}, s\right)\right]^{2}\right\} d r
\end{aligned}
$$

$$
\begin{aligned}
\left\langle\frac{s^{7 / 2}}{(1-s)^{4}}\right\rangle= & N_{n l}^{\prime 2} \int_{0}^{+\infty}\left\{\frac{s^{7 / 2}}{(1-s)^{4}} s^{2 \sqrt{\Lambda_{n l}}}\right. \\
& \cdot(1-s)^{2 \chi_{n l}}\left[2 F_{1}(-n, n\right. \\
& +2 \sqrt{\Lambda_{n l}}+2 \chi_{n l} ; 1 \\
& \left.\left.\left.+2 \sqrt{\Lambda_{n l}}, s\right)\right]^{2}\right\} d r \\
\left\langle\frac{s^{5 / 2}}{(1-s)^{4}}\right\rangle= & N_{n l}^{\prime 2} \int_{0}^{+\infty}\left\{\frac{s^{5 / 2}}{(1-s)^{4}} s^{2 \sqrt{\Lambda_{n l}}}\right. \\
& \cdot(1-s)^{2 \chi_{n l}}\left[2 F_{1}(-n, n\right. \\
& +2 \sqrt{\Lambda_{n l}}+2 \chi_{n l} ; 1 \\
& \left.\left.\left.+2 \sqrt{\Lambda_{n l}}, s\right)\right]^{2}\right\} d r
\end{aligned}
$$

with $N_{n l}^{\prime 2}=\left(\frac{\Gamma\left(n+2 \sqrt{\Lambda_{n l}}+1\right)}{n ! \Gamma\left(2 \sqrt{\Lambda_{n l}}+1\right)}\right)^{2} N_{n l}^{2}$ and we have used useful abbreviations $\langle n, l, m|X| n, l, m\rangle \equiv\langle X\rangle_{(n, l, m)}$ to avoid the extra burden of writing equations. Furthermore, we have applied the property of the spherical harmonics, which has the form $\iint Y_{l}^{m} Y_{l^{\prime}}^{m^{\prime}} \sin (\theta) d \theta d \varphi=\delta_{l l^{\prime}} \delta_{m m^{\prime}}$. We have $s=e^{-2 \alpha r}$, this allows us to obtain $d r=-\frac{1}{2 \alpha} \frac{d s}{s}$. From the asymptotic behavior of $s$ when $(r \rightarrow 0)(s \rightarrow+1)$ and when $(r \rightarrow+\infty)$ $(s \rightarrow 0)$, this allows reformulating Eqs. $(47, i=\overline{1,6})$ as follows:

$$
\begin{aligned}
& \left\langle\frac{s^{2}}{(1-s)^{4}}\right\rangle_{(n l m)}=\frac{N_{n l}^{\prime 2}}{2 \alpha} \int_{0}^{+1}\left\{s^{2 \sqrt{\Lambda_{n l}}+1}\right. \\
& \cdot(1-s)^{2 \chi_{n l}-4}\left[2 F_{1}(-n, n\right. \\
& +2 \sqrt{\Lambda_{n l}}+2 \chi_{n l} ; 1 \\
& \left.\left.\left.+2 \sqrt{\Lambda_{n l}}, s\right)\right]^{2}\right\} d s \\
& \left\langle\frac{s^{3 / 2}}{(1-s)^{3}}\right\rangle_{(n l m)}=\frac{N_{n l}^{\prime 2}}{2 \alpha} \int_{0}^{+1}\left\{s^{2 \sqrt{\Lambda_{n l}}+1 / 2}\right. \\
& \text { - }(1-s)^{2 \chi_{n l}-3}\left[2 F_{1}(-n, n\right. \\
& +2 \sqrt{\Lambda_{n l}}+2 \chi_{n l} ; 1 \\
& \left.\left.\left.+2 \sqrt{\Lambda_{n l}}, s\right)\right]^{2}\right\} d s \\
& \left\langle\frac{s^{5 / 2}}{(1-s)^{3}}\right\rangle_{(n l m)}=\frac{N_{n l}^{\prime 2}}{2 \alpha} \int_{0}^{+1}\left\{s^{2 \sqrt{\Lambda_{n l}}+3 / 2}\right. \\
& \cdot(1-s)^{2 \chi_{n l}-3}\left[2 F_{1}(-n, n\right. \\
& +2 \sqrt{\Lambda_{n l}}+2 \chi_{n l} ; 1 \\
& \left.\left.\left.+2 \sqrt{\Lambda_{n l}}, s\right)\right]^{2}\right\} d s \\
& \left\langle\frac{s^{3 / 2}}{(1-s)^{4}}\right\rangle_{(n l m)}=\frac{N_{n l}^{\prime 2}}{2 \alpha} \int_{0}^{+1}\left\{s^{2 \sqrt{\Lambda_{n l}}+1 / 2}\right. \\
& \text { - }(1-s)^{2 \chi_{n l}-4}\left[2 F_{1}(-n, n\right. \\
& +2 \sqrt{\Lambda_{n l}}+2 \chi_{n l} ; 1 \\
& \left.\left.\left.+2 \sqrt{\Lambda_{n l}}, s\right)\right]^{2}\right\} d s
\end{aligned}
$$




$$
\begin{aligned}
\left\langle\frac{s^{7 / 2}}{(1-s)^{4}}\right\rangle= & \frac{N_{n l}^{\prime 2}}{2 \alpha} \int_{0}^{+1}\left\{s^{2 \sqrt{\Lambda_{n l}}+5 / 2}\right. \\
& \cdot(1-s)^{2 \chi_{n l}-4}\left[2 F_{1}(-n, n\right. \\
& +2 \sqrt{\Lambda_{n l}}+2 \chi_{n l} ; 1 \\
& \left.\left.\left.+2 \sqrt{\Lambda_{n l}}, s\right)\right]^{2}\right\} d s \\
\left\langle\frac{s^{5 / 2}}{(1-s)^{4}}\right\rangle= & N_{n l}^{\prime 2} \int_{0}^{+1}\left\{s^{2 \sqrt{\Lambda_{n l}}+3 / 2}\right. \\
& \cdot(1-s)^{2 \chi_{n l}-4}\left[2 F_{1}(-n, n\right. \\
& +2 \sqrt{\Lambda_{n l}}+2 \chi_{n l} ; 1 \\
& \left.\left.\left.+2 \sqrt{\Lambda_{n l}}, s\right)\right]^{2}\right\} d s
\end{aligned}
$$

We can use the method proposed by Dong et al. ${ }^{73}$ and applied by Zhang, ${ }^{74}$ we calculate the integrals in Eqs. $(48, i=\overline{1,6})$. With the help of the special integral formula

$$
\begin{aligned}
& \left.\int_{0}^{+1} s^{\xi-1}(1-s)^{\sigma-1}{ }_{2} F_{1}\left(c_{1}, c_{2} ; c_{3} ; s\right)\right]^{2} d s \\
& =\frac{\Gamma(\xi) \Gamma(\sigma)}{\Gamma(\xi+\sigma)}{ }_{3} F_{2}\left(c_{1}, c_{2}, \sigma ; c_{3}, \sigma+\xi ; 1\right)
\end{aligned}
$$

here $3 F_{2}\left(c_{1}, c_{2}, \sigma ; c_{3}, \sigma+\xi ; 1\right)$ Is obtained from the generalized hypergeometric function $3 F_{2}\left(\alpha_{1}, \alpha_{2}, \ldots, \alpha_{p} ; \beta_{1}, \beta_{2}, \ldots\right.$, $\left.\beta_{1} ; 1\right)$ for $p=3$ and $1=2$ while $\Gamma(\sigma)$ denoting the usual Gamma function. We obtain from Eqs. $(48, i=\overline{1,6})$ the following results:

$$
\begin{aligned}
& \left\langle\frac{s^{2}}{(1-s)^{4}}\right\rangle_{(n l m)} \\
& =\frac{N_{n l}^{\prime 2}}{2 \alpha} \frac{\Gamma\left(2 \sqrt{\Lambda_{n l}}+2\right) \Gamma\left(2 \chi_{n l}-3\right)}{\Gamma\left(2 \sqrt{\Lambda_{n l}}+2 \chi_{n l}-1\right)} \\
& { }_{3} F_{2}\left(-n, n+2 \sqrt{\Lambda_{n l}}+2 \chi_{n l}, 2 \chi_{n l}-3\right. \text {; } \\
& \left.1+2 \sqrt{\Lambda_{n l}}, 2 \chi_{n l}+2 \sqrt{\Lambda_{n l}}-1 ; 1\right) \\
& \left\langle\frac{s^{3 / 2}}{(1-s)^{3}}\right\rangle_{(n l m)} \\
& =\frac{N_{n l}^{\prime 2}}{2 \alpha} \frac{\Gamma\left(2 \sqrt{\Lambda_{n l}}+3 / 2\right) \Gamma\left(2 \chi_{n l}-2\right)}{\Gamma\left(2 \sqrt{\Lambda_{n l}}+2 \chi_{n l}-1 / 2\right)} \\
& \cdot{ }_{3} F_{2}\left(-n, n+2 \sqrt{\Lambda_{n l}}+2 \chi_{n l}, 2 \chi_{n l}-2\right. \text {; } \\
& \left.1+2 \sqrt{\Lambda_{n l}}, 2 \chi_{n l}+2 \sqrt{\Lambda_{n l}}-1 / 2 ; 1\right) \\
& \left\langle\frac{s^{5 / 2}}{(1-s)^{3}}\right\rangle_{(n l m)} \\
& =\frac{N_{n l}^{\prime 2}}{2 \alpha} \frac{\Gamma\left(2 \sqrt{\Lambda_{n l}}+5 / 2\right) \Gamma\left(2 \chi_{n l}-2\right)}{\Gamma\left(2 \sqrt{\Lambda_{n l}}+2 \chi_{n l}+1 / 2\right)} \\
& { }_{3} F_{2}\left(-n, n+2 \sqrt{\Lambda_{n l}}+2 \chi_{n l}, 2 \chi_{n l}-2\right. \text {; } \\
& \left.1+2 \sqrt{\Lambda_{n l}}, 2 \chi_{n l}+2 \sqrt{\Lambda_{n l}}+1 / 2 ; 1\right) \\
& \left\langle\frac{s^{3 / 2}}{(1-s)^{4}}\right\rangle_{(n l m)} \\
& =\frac{N_{n l}^{\prime 2}}{2 \alpha} \frac{\Gamma\left(2 \sqrt{\Lambda_{n l}}+3 / 2\right) \Gamma\left(2 \chi_{n l}-3\right)}{\Gamma\left(2 \sqrt{\Lambda_{n l}}+2 \chi_{n l}-3 / 2\right)} \\
& \cdot{ }_{3} F_{2}\left(-n, n+2 \sqrt{\Lambda_{n l}}+2 \chi_{n l}, 2 \chi_{n l}-3\right. \text {; } \\
& \left.1+2 \sqrt{\Lambda_{n l}}, 2 \chi_{n l}+2 \sqrt{\Lambda_{n l}}-3 / 2 ; 1\right)
\end{aligned}
$$

$$
\begin{aligned}
&\left\langle\frac{s^{7 / 2}}{(1-s)^{4}}\right\rangle_{(n l m)} \frac{N_{n l}^{\prime 2}}{2 \alpha} \frac{\Gamma\left(2 \sqrt{\Lambda_{n l}}+5 / 2\right) \Gamma\left(2 \chi_{n l}-3\right)}{\Gamma\left(2 \sqrt{\Lambda_{n l}}+2 \chi_{n l}-1 / 2\right)} \\
&={ }_{3} F_{2}\left(-n, n+2 \sqrt{\Lambda_{n l}}+2 \chi_{n l}, 2 \chi_{n l}-3 ;\right. \\
&\left.1+2 \sqrt{\Lambda_{n l}}, 2 \chi_{n l}+2 \sqrt{\Lambda_{n l}}-1 / 2 ; 1\right) \\
&\left\langle\frac{s^{5 / 2}}{(1-s)^{4}}\right\rangle \\
&=\frac{N_{n l}^{\prime 2}}{2 \alpha} \frac{\Gamma\left(2 \sqrt{\Lambda_{n l}}+5 / 2\right) \Gamma\left(2 \chi_{n l}-3\right)}{\Gamma\left(2 \sqrt{\Lambda_{n l}}+2 \chi_{n l}-1 / 2\right)} \\
& \cdot{ }_{3} F_{2}\left(-n, n+2 \sqrt{\Lambda_{n l}}+2 \chi_{n l}, 2 \chi_{n l}-3 ;\right. \\
&\left.1+2 \sqrt{\Lambda_{n l}}, 2 \chi_{n l}+2 \sqrt{\Lambda_{n l}}-1 / 2 ; 1\right)
\end{aligned}
$$

\subsection{THE ENERGY SHIFT FOR THE IMSPM IN RNCQM SYMMETRIES}

The global relativistic energy shift for the improved modified Mobius squared potential model in RNCQM symmetries is composed of three principal parts. The first one is produced from the effect of the generated spin-orbit effective potential. This effective potential is obtained by replacing the coupling of the angular momentum operator and the noncommutative vector $L \Theta$ with the new equivalent coupling $\Theta \mathbf{L S}$ (with $\Theta^{2}=\Theta_{12}^{2}+\Theta_{23}^{2}+\Theta_{13}^{2}$ ). This degree of freedom comes considering that the infinitesimal noncommutative vector $\Theta$ is arbitrary. We have chosen it to a parallel of the spin $S$ of the diatomic molecules under modified Mobius squared potential. Furthermore, we replace the new spin-orbit coupling $\Theta L S$ with the corresponding physical form $(\Theta / 2) G^{2}, G^{2}=J^{2}-L^{2}-S^{2}$. Moreover, in quantum mechanics, the operators $\left(H_{r n c}^{m s p}, J^{2}, L^{2}, S^{2}\right.$ and $\left.J_{z}\right)$ forms a complete set of conserved physics quantities, the eigenvalues of the operator $G^{2}$ are equal to the values $f(j, l, s)=$ $[j(j+1)-l(l+1)-s(s+1)] / 2$, with $|l-s| \leq j \leq|l+s|$. As a direct consequence, the partial energy shift $\Delta E_{m s p}^{s o}\left(n, \alpha, A, B, V_{0}, \Theta, j, l, s\right)$ due to the perturbed effective potential $V_{\text {pert }}^{m s p}(s)$ produced for the $n^{\text {th }}$ excited state, in DRKGT symmetries as follows:

$$
\begin{aligned}
& \Delta E_{m s p}^{s o}\left(n, \alpha, A, B, V_{0}, \Theta, j, l, s\right) \\
& =\langle\Xi\rangle_{(n l m)}^{R m s p}\left(n, \alpha, A, B, V_{0}\right) \\
& \quad \cdot \Theta(j(j+1)-l(l+1)-s(s+1))
\end{aligned}
$$

The global expectation value $\langle\Xi\rangle_{(n l m)}^{R m s p}\left(n, \alpha, A, B, V_{0}\right)$ is determined from the following expression: 


$$
\begin{aligned}
\langle\Xi\rangle_{(n l m)}^{R m s p}\left(n, \alpha, A, B, V_{0}\right) & 16 l(l+1) \alpha^{4}\left\langle\frac{s^{2}}{(1-s)^{4}}\right\rangle_{(n l m)} \\
& -4\left(E_{n l}+M\right) \alpha^{2} V_{0}\left(A B\left\langle\frac{s^{3 / 2}}{(1-s)^{3}}\right\rangle_{(n l m)}\right. \\
& +B^{2}\left\langle\frac{s^{5 / 2}}{(1-s)^{3}}\right\rangle_{(n l m)}-A^{2}\left\langle\frac{s^{3 / 2}}{(1-s)^{4}}\right\rangle_{(n l m)} \\
& -B^{2}\left\langle\frac{s^{7 / 2}}{(1-s)^{4}}\right\rangle_{(n l m)} \\
& \left.-2 A B\left\langle\frac{s^{5 / 2}}{(1-s)^{4}}\right\rangle_{(n l m)}\right)
\end{aligned}
$$

The second part is obtained from the magnetic effect of the perturbative effective potential $V_{\text {pert }}^{m s p}(s)$ under the improved modified Mobius squared potential model. This effective potential is achieved when we replace both $(L \Theta$ and $\left.\Theta_{12}\right)$ by $\left(\sigma \aleph L_{z}\right.$ and $\left.\sigma \aleph\right)$, respectively, here ( $\aleph$ and $\sigma$ ) symbolize the intensity of the magnetic field induced by the effect of the deformation of space-space geometry and a new infinitesimal noncommutativity parameter, so that the physical unit of the original noncommutativity parameter $\Theta_{12}$ (length) $)^{2}$ is the same unit of $\sigma \aleph$, we have also need to apply $\left\langle n^{\prime}, l^{\prime}, m^{\prime} L_{z} n, l, m\right\rangle=m \delta_{m^{\prime} m} \delta_{l^{\prime} l} \delta_{n^{\prime} n}\left(-l^{\prime} \leq m^{\prime} \leq l\right.$ and $-l \leq m \leq l)$. All of this data allows for the discovery of the new energy shift $\Delta E_{m s p}^{m a g}\left(n, \alpha, A, B, V_{0}, \sigma, m\right)$ due to the perturbed Zeeman effect which created by the influence of the improved modified Mobius squared potential model for the $n^{\text {th }}$ excited-state in RNCQM symmetries as follows:

$$
\begin{aligned}
& \Delta E_{m s p}^{m a g}\left(n, \alpha, A, B, V_{0}, \sigma, j, l, s\right) \\
& =\sigma \aleph\langle\Xi\rangle_{(n l m)}^{R m s p}\left(n, \alpha, A, B, V_{0}\right) m
\end{aligned}
$$

Now, for our purposes, we are interested in finding a new third automatically important symmetry for the improved modified Mobius squared potential model at zero temperature in DRKGT symmetries. This physical phenomenon is induced automatically from the influence of a perturbed effective potential $V_{p e r t}^{m s p}(s)$ which we have seen in Eq. (46). We discover these important physical phenomena when our studied system consists of non-interacting is considered as Fermi gas, it is formed from all the particles in their gaseous state $\left(\mathrm{H}_{2}, \mathrm{I}_{2}, \mathrm{CO}, \mathrm{NO}\right.$, and $\left.\mathrm{HCl}\right)$ undergoing rotation with angular velocity $\Omega$ if we make the following two simultaneous transformations to ensure that the previous calculations are not repeated:

$$
\Theta \rightarrow \chi \Omega \text { and } \mathbf{L} \Theta \rightarrow \chi \mathbf{L} \boldsymbol{\Omega}
$$

Here $\chi$ is just infinitesimal real proportional constants. We can express the effective potential $V_{p e r t}^{m s p-r o t}(s)$ which induced the rotational movements of the diatomic molecules as follows:

$$
\begin{aligned}
V_{p e r t}^{m s p-r o t}(s)= & \frac{16 l(l+1) \alpha^{4} s^{2}}{(1-s)^{4}} \chi L \Omega \\
& -4\left(E_{n l}+M\right) \alpha^{2}\left(\frac{V_{0} A B s^{3 / 2}}{(1-s)^{3}}\right. \\
& +\frac{V_{0} B^{2} s^{5 / 2}}{(1-s)^{3}}-\frac{V_{0} A^{2} s^{3 / 2}}{(1-s)^{4}} \\
& \left.-\frac{V_{0} B^{2} s^{7 / 2}}{(1-s)^{4}}-\frac{2 V_{0} A B s^{5 / 2}}{(1-s)^{4}}\right) \chi \mathbf{L} \boldsymbol{\Omega}
\end{aligned}
$$

To simplify the calculations without compromising physical content, we choose the rotational velocity $\Omega$ parallel to the $(O z)$ axis. Then we transform the spin-orbit coupling to the new physical phenomena as follows:

$$
V(s) L \Omega=\chi V(s) \Omega L_{z}
$$

With

$$
\begin{aligned}
& V(s) \frac{16 l(l+1) \alpha^{4} s^{2}}{(1-s)^{4}} \\
& -4\left(E_{n l}+M\right) \alpha^{2}\left(\frac{V_{0} A B s^{3 / 2}}{(1-s)^{3}}\right. \\
& +\frac{V_{0} B^{2} s^{5 / 2}}{(1-s)^{3}}-\frac{V_{0} A^{2} s^{3 / 2}}{(1-s)^{4}} \\
& \left.-\frac{V_{0} B^{2} s^{7 / 2}}{(1-s)^{4}}-\frac{2 V_{0} A B s^{5 / 2}}{(1-s)^{4}}\right)
\end{aligned}
$$

All of this data allows for the discovery of the new energy shift $\Delta E_{m s p}^{f-r o t}\left(n, \alpha, A, B, V_{0}, \chi, m\right)$ due to the perturbed Fermi gas effect $V_{\text {pert }}^{\text {msp-rot }}(r)$ which generated automatically by the influence of the modified Mobius squared potential for the $n^{\text {th }}$ excited-state in DRKGT symmetries as follows:

$$
\begin{aligned}
& \Delta E_{m s p}^{f-r o t}\left(n, \alpha, A, B, V_{0}, \chi, m\right) \\
& =\chi\langle\Xi\rangle_{(n l m)}^{R m s p}\left(n, \alpha, A, B, V_{0}, V_{2}\right) \Omega m
\end{aligned}
$$

It is worth mentioning that Bencheikh et al. ${ }^{75}$ and Medjedel and Bencheikh ${ }^{76}$ studied rotating isotropic and anisotropic harmonically confined ultra-cold Fermi gas in a two and three-dimensional space at zero temperature, but in this study, the rotational term was added to the Hamiltonian operator, in contrast to our case, where this rotation term $\chi V(s) L \Omega$ automatically appears due to the large symmetries resulting from the deformation of space-phase.

\subsection{THE SPECTRUM OF RELATIVISTIC DKGT UNDER IMSPM}

In this section of the paper, we summarize our obtained results $\Delta E_{m s p}^{s o}\left(n, \alpha, A, B, V_{0}, j, l, s\right), \Delta E_{m s p}^{m a g}\left(n, \alpha, A, B, V_{0}, m\right)$ and $\Delta E_{m s p}^{f-r o t}\left(n, \alpha, A, B, V_{0}, m\right)$ for the $n^{\text {th }}$ excited-state due to the spin-orbital coupling modified Zeeman effect and perturbed Fermi gas potential induced by $V_{p e r t}^{m s p}(s)$ on based on the superposition principle in the deformed relativistic Klien-Gordon theory under the improved modified Mobius squared potential model. This allows us to deduce the additive energy shift $\Delta E_{m s p}^{\text {tot }}\left(n, \alpha, A, B, V_{0}, j, l, s, m\right)$ under the influence of the improved modified Mobius squared potential model in RNCQM symmetries as follows

$$
\begin{aligned}
\Delta & E_{m s p}^{t o t}\left(n, \alpha, A, B, V_{0}, \Theta, \sigma, \chi, j, l, s, m\right) \\
= & \langle\Xi\rangle_{(n l m)}^{R m s p}\left(n, \alpha, A, B, V_{0}\right)(\Theta f(j, l, s) \\
& +\sigma \aleph m+\chi \Omega m)
\end{aligned}
$$

The above results present the global energy shift, which was generated with the effect of noncommutativity properties of space-space; it depended explicitly on the noncommutativity parameters $(\Theta, \sigma, \chi)$, the parameters of the modified Mobius square potential $\left(\alpha, A, B, V_{0}\right)$ in addition to the atomic quantum numbers $(j, l, s, m)$. We observed that the obtained global effective energy under the modified Mobius squared potential has a carry unit of energy because it is combined with the carrier of energy $\left(M^{2}-E_{n l}^{2}\right)$. As a direct consequence, the energy $E_{r-n c}^{m s p}\left(n, \alpha, A, B, V_{0}, j, l, s, m\right)$ produced with the improved modified Mobius squared potential model, in the symmetries of RNCQM is the sum 
of the root quart of the shift energy $\Delta\left[E_{m s p}^{f-r o t}\left(n, \alpha, A, B, V_{0}, \chi, m\right)\right]^{1 / 2}$ and the relativist energy $E_{n l}$ produced by the effect due to the effect of the modified Mobius squared potential in RQM, as follows:

$$
\begin{gathered}
E_{r-n c}^{m s p}\left(n, \alpha, A, B, V_{0}, \Theta, \sigma, \chi, j, l, s, m\right) \\
=E_{n l}+\left[\langle\Xi\rangle_{(n l m)}^{R m s p}\left(n, \alpha, A, B, V_{0}\right)\right. \\
\cdot(\Theta f(j, l, s)+\sigma \aleph m+\chi \Omega m)]^{1 / 2}
\end{gathered}
$$

The relativist energy $E_{n l}$ is determined from the energy equation (28). For the ground state and first excited state, the above equation can be reduced to the following form:

$$
\begin{gathered}
E_{r-n c}^{m s p}\left(n=0, \alpha, A, B, V_{0}, \Theta, \sigma, \chi, j, l, s, m\right) \\
=E_{0 l}+\left[\langle\Xi\rangle_{(n l m)}^{R m s p}\left(n=0, \alpha, A, B, V_{0}\right)\right. \\
\cdot(\Theta f(j, l, s)+\sigma \aleph m+\chi \Omega m)]^{1 / 2}
\end{gathered}
$$

and

$$
\begin{gathered}
E_{r-n c}^{m s p}\left(n=1, \alpha, A, B, V_{0}, \Theta, \sigma, \chi, j, l, s, m\right) \\
=E_{1 l}+\left[\langle\Xi\rangle_{(n l m)}^{R m s p}\left(n=1, \alpha, A, B, V_{0}\right)\right. \\
\cdot(\Theta f(j, l, s)+\sigma \aleph m+\chi \Omega m)]^{1 / 2}
\end{gathered}
$$

Eq. (62) can de describe the relativistic energy of some diatomic molecules such as $\mathrm{H}_{2}, \mathrm{I}_{2}$, $\mathrm{CO}, \mathrm{NO}$, and $\mathrm{HCl}$ under the improved modified Mobius squared potential model in the DRKGT symmetries.

\subsection{RELATIVISTIC PARTICULAR CASES UNDER THE IMSPM IN THE DRKGT SYMMETRIES}

We now consider a special case of interest of the modified Mobius square potential, known by the Hua potential, the Deng-Fan potential is given by ${ }^{77}$ :

$$
V_{d f}(r)=D_{e} \frac{1-2(1+b) e^{-\alpha r}+(1+b)^{2} e^{-2 \alpha r}}{\left(1-e^{-\alpha r}\right)^{2}}
$$

Comparing Eq. (63) with the modified Mobius square potential in Eq. (11), we get:

$$
\begin{gathered}
A^{2} \mapsto \frac{D_{e}}{V_{0}, A B} \mapsto-\frac{D_{e}(1+b)}{V_{0},}, \\
B^{2} \mapsto D_{e}(1+b)^{2} / V_{0} \text { and } 2 \alpha \mapsto \alpha
\end{gathered}
$$

The perturbed effective potential $V_{\text {pert }}^{m s p}(s)$ in Eq. (46) turns to the perturbed effective potential $V_{\text {pert }}^{d f}(s)$ in the symmetries of RNCQM as follows:

$$
\begin{aligned}
V_{\text {pert }}^{d f}(s)= & \left(\frac{l(l+1) \alpha^{4} s^{2}}{(1-s)^{4}}-\left(E_{n l}+M\right) \alpha^{2}\right. \\
& \cdot\left(-\frac{(1+b) s^{3 / 2}}{(1-s)^{3}}+\frac{(1+b)^{2} s^{5 / 2}}{(1-s)^{3}}\right. \\
& -\frac{s^{3 / 2}}{(1-s)^{4}}-\frac{(1+b)^{2} s^{7 / 2}}{(1-s)^{4}} \\
& \left.\left.+\frac{2(1+b) s^{5 / 2}}{(1-s)^{4}}\right)\right) D_{e} \mathbf{L} \Theta+O\left(\Theta^{2}\right)
\end{aligned}
$$

In this case, the additive energy shift $\Delta E_{d f}^{t o t}\left(n, \alpha, D_{e}, b, j, l, s, m\right)$ under the influence of the modified equally mixed deformed generalized Deng-Fan potential in DRKGT symmetries is determined from the following formula:

$$
\begin{aligned}
\Delta & E_{d f}^{t o t}\left(n, \alpha, D_{e}, b, V_{0}, \Theta, \sigma, \chi, j, l, s, m\right) \\
= & \langle\Xi\rangle_{(n l m)}^{R d f}\left(n, \alpha, D_{e}, b\right) \\
& \cdot(\Theta f(j, l, s)+\sigma \aleph+m \chi \Omega m)
\end{aligned}
$$

Thus, the corresponding global expectation value $\langle\Xi\rangle_{(n l m)}^{R d f}\left(n, \alpha, D_{e}, b, V_{0}\right)$ is determined from the following expression:

$$
\begin{aligned}
\langle\Xi\rangle_{(n l m)}^{R d f}\left(n, \alpha, D_{e}, b\right) & l(l+1) \alpha^{4}\left\langle\frac{s^{4}}{(1-s)^{4}}\right\rangle_{(n l m)} \\
& -D_{e} \alpha^{2}\left(E_{n l}+M\right) \\
& \cdot\left(-(1+b)\left\langle\frac{s^{\frac{3}{2}}}{(1-s)^{3}}\right\rangle_{(n l m)}\right. \\
& +(1+b)^{2}\left\langle\frac{s^{\frac{5}{2}}}{(1-s)^{3}}\right\rangle_{(n l m)} \\
& -\left\langle\frac{s^{\frac{3}{2}}}{(1-s)^{4}}\right\rangle_{(n l m)} \\
& -(1+b)^{2}\left\langle\frac{s^{\frac{7}{2}}}{(1-s)^{4}}\right\rangle_{(n l m)} \\
& \left.+2(1+b)\left\langle\frac{s^{\frac{5}{2}}}{(1-s)^{4}}\right\rangle_{(n l m)}\right)
\end{aligned}
$$

The new relativistic energy in Eq. (62) reduces to the new energy $E_{r-n c}^{d f}\left(n, \alpha, D_{e}, b, \Theta, \sigma, \chi, j, l, s, m\right)$ under new deformed generalized Deng-Fan potential in DRKGT symmetries, as follows:

$$
\begin{aligned}
& E_{r-n c}^{d f}\left(n, \alpha, D_{e}, b, \Theta, \sigma, \chi, j, l, s, m\right) \\
& =E_{n l}^{d p}+\left[\langle\Xi\rangle_{(n l m)}^{R d f}\left(n, \alpha, D_{e}, b, V_{0}\right)\right. \\
& \cdot(\Theta f(j, l, s)+\sigma \aleph m+m \chi \Omega m)]^{1 / 2}
\end{aligned}
$$

Making the corresponding parameter replacements in Eq. (28), we obtain the energy equation for the deformed generalized Deng-Fan potential in the Klein-Gordon theory with equally mixed potentials, in RQM symmetries as:

$$
\begin{aligned}
& E_{n l}^{2}-M^{2} \\
& =-D_{e}\left(E_{n l}+M\right)-4 \alpha^{2} \\
& \cdot\left[\frac{\frac{D_{e} b\left(E_{n l}+M\right)}{2 \alpha^{2}}+1 / 2+n(n+1)}{1+2 n+\sqrt{(2 l+1)^{2}-\frac{D_{e}(2+b)^{2}\left(E_{n l}+M\right)}{2 \alpha^{2}}}}\right] \\
& -4 \alpha^{2} \\
& \cdot\left[\frac{(n+1 / 2) \sqrt{(2 l+1)^{2}-\frac{D_{e}(2+b)^{2}\left(E_{n l}+M\right)}{2 \alpha^{2}}}}{1+2 n+\sqrt{(2 l+1)^{2}-\frac{D_{e}(2+b)^{2}\left(E_{n l}+M\right)}{2 \alpha^{2}}}}\right]
\end{aligned}
$$

\section{FERMIONIC MASSIVE SPIN-1/2 PARTICLES INTERACTING WITH THE RELATIVISTIC IMSPM IN THE DEFORMED DIRAC THEORY SYMMETRIES}

To obtain the improved Dirac equation MDE for the improved modified Mobius square potential model in the symmetries of deformed Dirac theory (DDT), we replace both the ordinary Hamiltonian operators $H\left(p_{i}, x_{i}\right)$, ordinary spinors $\Psi_{n k}(r, \theta, \phi)$, and ordinary energy $E_{n k}$ by NC Hamiltonian operator $H\left(p_{n c i}, x_{n c i}\right)$, the new spinor $\Psi_{n k}\left(\overleftrightarrow{r}_{n c}\right)$, and new energy $E_{n c-n k}^{m p}$ and the ordinary product will be replaced by star product $*$, respectively. This allows us to write the MED for improved modified Mobius square poten- 
tial as follows ${ }^{67-70}$ :

$$
H\left(p_{n c i}, x_{n c i}\right) * \Psi_{n k}\left(\vec{r}_{n c}\right)=E_{n c-n k}^{m p} \Psi_{n k}\left(\vec{r}_{n c}\right)
$$

It is worth motioning that Bopp's shift method permutes to reduce the above equation to the simplest form:

$$
H_{m p}\left(p_{n c i}, x_{n c i}\right) \Psi_{n k}(\vec{r})=E_{n c-n k}^{m p} \Psi_{n k}(\vec{r})
$$

The new Hamiltonian operator for fermionic massive spin $1 / 2$ particles interacting with the relativistic improved Hellmann-generalized Morse potential model is given by:

$$
\begin{aligned}
H_{m p}\left(p_{n c i}, x_{n c i}\right)= & \alpha p_{n c}+\beta\left(M+S_{m s p}\left(r_{n c}\right)\right) \\
& +V_{m s p}\left(r_{n c}\right)
\end{aligned}
$$

By applying the notion of the Weyl Moyal star product which has been seen previously in Eqs. (2), (3), and (4), the differential equations that are satisfied by the radial wave function in Eqs. (22) and (23), for the spin symmetry solutions and pseudospin symmetry of MDE with improved modified Mobius square potential, respectively, in the deformed Dirac theory symmetries, become as follows:

$$
\left[\begin{array}{c}
\frac{d^{2}}{d r^{2}}-\frac{k(k+1)}{r_{n c}^{2}}+\frac{2 k U\left(r_{n c}\right)}{r_{n c}} \\
-\frac{d U\left(r_{n c}\right)}{d r}-U^{2}\left(r_{n c}\right) \\
-\left(M+E_{n k}^{s}-C_{s}\right) \\
\cdot\left(M-E_{n k}^{s}-V_{0}\left(\frac{A+B e^{-2 \alpha r_{n c}}}{1-e^{-2 \alpha r_{n c}}}\right)^{2}\right)
\end{array}\right] F_{n k}(r)=0
$$

And by applying the relationship recorded in Eq. (1), and by looking at the result of Eq. (43) we find:

$$
\begin{aligned}
& V_{m s p}\left(r_{n c}\right) \\
& =V_{m s p}(r) \\
& -\left(\begin{array}{c}
\frac{2 V_{0} \alpha B A e^{-2 a r}}{r\left(1-e^{-2 a r}\right)^{2}}+\frac{2 V_{0} \alpha B^{2} e^{-4 a r}}{r\left(1-e^{-2 a r}\right)^{2}} \\
-\frac{2 V_{0} \alpha A^{2} e^{-2 a r}}{r\left(1-e^{-2 a r}\right)^{3}}-\frac{2 V_{0} \alpha B^{2} e^{-6 a r}}{r\left(1-e^{-2 a r}\right)^{3}} \\
-\frac{4 V_{0} A B \alpha e^{-4 a r}}{r\left(1-e^{-2 a r}\right)^{3}}
\end{array}\right) \mathbf{L} \boldsymbol{\Theta} \\
& ++O\left(\Theta^{2}\right) \\
& \frac{2 k U\left(r_{n c}\right)}{r_{n c}}=\frac{2 k U(r)}{r}-\frac{\partial}{\partial r}\left(\frac{2 k U(r)}{r}\right) \frac{\mathbf{L} \Theta}{2 r} \\
& +O\left(\Theta^{2}\right) \\
& \frac{d U\left(r_{n c}\right)}{d r}=\frac{d U(r)}{d r}-\frac{\partial}{\partial r}\left(\frac{d U(r)}{d r}\right) \frac{\mathbf{L} \Theta}{2 r} \\
& +O\left(\Theta^{2}\right) \\
& U^{2}\left(r_{n c}\right)=U^{2}(r)-\frac{\partial}{\partial r}\left(U^{2}(r)\right) \frac{\mathbf{L} \Theta}{2 r}+O\left(\Theta^{2}\right)
\end{aligned}
$$

here $U(r)$ is the Yukawa tensor interaction. ${ }^{3}$ After a straightforward calculation, we find:

$$
\begin{aligned}
& -\frac{2 k U\left(r_{n c}\right)}{r_{n c}}=-\frac{2 k U(r)}{r} \\
& -k V_{1}\left(\frac{\alpha e^{-\alpha r}}{r^{3}}+\frac{2 e^{-\alpha r}}{r^{4}}\right) \\
& \cdot L \Theta+O\left(\Theta^{2}\right) \\
& \frac{d U\left(r_{n c}\right)}{d r}=\frac{d U(r)}{d r} \\
& +\frac{V_{1}}{2}\left(\begin{array}{c}
\frac{\alpha^{2} e^{-\alpha r}}{r^{2}}+\frac{\alpha e^{-\alpha r}}{r^{3}} \\
+\frac{\alpha e^{-\alpha r}}{r^{3}}+\frac{2 e^{-\alpha r}}{r^{4}}
\end{array}\right) \\
& \cdot \mathbf{L} \Theta+O\left(\Theta^{2}\right) \\
& U^{2}\left(r_{n c}\right)=U^{2}(r)+V_{1}^{2}\left(\frac{\alpha e^{-2 \alpha r}}{r^{3}}+\frac{e^{-2 \alpha r}}{r^{4}}\right) \\
& \cdot \mathbf{L} \Theta+O\left(\Theta^{2}\right) \\
& \left\{\begin{array}{l}
\frac{k(k-1)}{r_{n c}^{2}}=\frac{k(k-1)}{r^{2}}+\frac{k(k-1)}{r^{4}} \mathbf{L} \Theta+O\left(\Theta^{2}\right) \\
\frac{k(k+1)}{r_{n c}^{2}}=\frac{k(k+1)}{r^{2}}+\frac{k(k+1)}{r^{4}} \mathbf{L} \Theta+O\left(\Theta^{2}\right)
\end{array}\right.
\end{aligned}
$$

And by inserting Eqs. (82), (81), (80), (79), (78), (77), and (75) into the two expressions of the equations (73) and (74), we have obtained:

$$
\begin{gathered}
{\left[\frac{d^{2}}{d r^{2}}-\frac{k(k+1)}{r^{2}}+\frac{2 k U(r)}{r}-\frac{d U(r)}{d r}\right.} \\
-U^{2}(r)-\left(M+E_{n k}-C_{s}\right) \\
\cdot\left(M-E_{n k}-V_{0}\left(\frac{A+B e^{-2 \alpha r}}{1-e^{-2 \alpha r}}\right)^{2}\right) \\
\left.\quad+V_{p e r t}^{s p-s y}(r)\right] F_{n k}(r)=0 \\
{\left[\frac{d^{2}}{d r^{2}}-\frac{k(k-1)}{r^{2}}+\frac{2 k U(r)}{r}-\frac{d U(r)}{d r}\right.} \\
\quad-U^{2}(r)-\left(M-E_{n k}+C_{p s}\right) \\
\cdot\left(M+E_{n k}+V_{0}\left(\frac{A+B e^{-2 \alpha r}}{1-e^{-2 \alpha r}}\right)^{2}\right) \\
\left.+V_{p e r t}^{p s p-s y}(r)\right] G_{n k}(r)=0
\end{gathered}
$$

with

$$
\begin{aligned}
V_{p e r t}^{s p-s y}(r)= & \frac{k(k+1)}{r^{4}} \\
& -k V_{1}\left(\frac{\alpha e^{-\alpha r}}{r^{3}}+\frac{2 e^{-\alpha r}}{r^{4}}\right) \\
& +V_{1}^{2}\left(\frac{\alpha e^{-2 \alpha r}}{r^{3}}+\frac{e^{-2 \alpha r}}{r^{4}}\right) \\
& +\left(M+E_{n k}-C_{s}\right) \\
& \cdot\left(\frac{2 V_{0} \alpha B A e^{-2 \alpha r}}{r\left(1-e^{-2 \alpha r}\right)^{2}}+\frac{2 V_{0} \alpha B^{2} e^{-4 \alpha r}}{r\left(1-e^{-2 \alpha r}\right)^{2}}\right. \\
& -\frac{2 V_{0} \alpha A^{2} e^{-2 \alpha r}}{r\left(1-e^{-2 \alpha r}\right)^{3}}-\frac{2 V_{0} \alpha B^{2} e^{-6 \alpha r}}{r\left(1-e^{-2 \alpha r}\right)^{3}} \\
& \left.\left.-\frac{4 V_{0} A B \alpha e^{-4 \alpha r}}{r\left(1-e^{-2 \alpha r}\right)^{3}}\right)\right] \mathbf{L \Theta}
\end{aligned}
$$

and 


$$
\begin{aligned}
V_{p e r t}^{p s p-s y}(r)= & \frac{k(k-1)}{r^{4}} \\
& -k V_{1}\left(\frac{\alpha e^{-\alpha r}}{r^{3}}+\frac{2 e^{-\alpha r}}{r^{4}}\right) \\
& +V_{1}^{2}\left(\frac{\alpha e^{-2 \alpha r}}{r^{3}}+\frac{e^{-2 \alpha r}}{r^{4}}\right) \\
& +\left(M-E_{n k}+C_{p s}\right) \\
& \cdot\left(\frac{2 V_{0} \alpha B A e^{-2 \alpha r}}{r\left(1-e^{-2 \alpha r}\right)^{2}}+\frac{2 V_{0} \alpha B^{2} e^{-4 \alpha r}}{r\left(1-e^{-2 \alpha r}\right)^{2}}\right. \\
& -\frac{2 V_{0} \alpha A^{2} e^{-2 \alpha r}}{r\left(1-e^{-2 \alpha r}\right)^{3}}-\frac{2 V_{0} \alpha B^{2} e^{-6 \alpha r}}{r\left(1-e^{-2 \alpha r}\right)^{3}} \\
& \left.\left.-\frac{4 V_{0} A B \alpha e^{-4 \alpha r}}{r\left(1-e^{-2 \alpha r}\right)^{3}}\right)\right] \mathbf{L \Theta}
\end{aligned}
$$

We apply the Greene and Aldrich approximation on the two perturbative effective of spin symmetry and pseudospin symmetry to the Eqs. (85) and (86) to obtain:

$$
\begin{aligned}
V_{p e r t}^{s p-s y}(s) & \left(\frac{16 \alpha^{4} k(k+1) s^{2}}{(1-s)^{4}}-\frac{2 k V_{1} \alpha^{2} s^{2}}{(1-s)^{3}}+\frac{16 V_{1}^{2} \alpha^{4} s^{2}}{(1-s)^{2}}\right. \\
& \left.+\frac{\left(4\left(M+E_{n k}-C_{s}\right) \alpha^{2} V_{0} B A+2 V_{1}^{2} \alpha^{2}\right) s^{3 / 2}}{(1-s)^{3}}\right) \\
& \cdot L \Theta+4\left(M+E_{n k}-C_{s}\right) \alpha^{2} \\
& -8 \alpha\left(V_{0} A B\left(M+E_{n k}-C_{s}\right) \alpha L \Theta+4 k V_{1}\right) \\
& \cdot \frac{s^{5 / 2}}{(1-s)^{4}}-8 \alpha\left(V_{0} A B\left(M+E_{n k}-C_{s}\right) \alpha+4 k V_{1}\right) \\
& \cdot \frac{s^{5 / 2}}{(1-s)^{4}} \mathbf{L} \boldsymbol{\Omega}
\end{aligned}
$$

and

$$
\begin{aligned}
& V_{\text {pert }}^{\text {psp-sy }}(s) \\
& =\left(\frac{16 \alpha^{4} k(k-1) s^{2}}{(1-s)^{4}}-\frac{2 k V_{1} \alpha^{2} s^{2}}{(1-s)^{3}}+\frac{16 V_{1}^{2} \alpha^{4} s^{2}}{(1-s)^{2}}\right. \\
& \left.\quad+\frac{\left(4\left(M-E_{n k}+C_{p s}\right) \alpha^{2} V_{0} B A+2 V_{1}^{2} \alpha^{2}\right) s^{3 / 2}}{(1-s)^{3}}\right) \\
& \quad \cdot L \Theta+4\left(M-E_{n k}+C_{p s}\right) \alpha^{2}\left(V_{0} B^{2} \frac{s^{5 / 2}}{(1-s)^{3}}\right. \\
& \left.\quad-V_{0} A^{2} \frac{s^{3 / 2}}{(1-s)^{4}}-V_{0} B^{2} \frac{s^{7 / 2}}{(1-s)^{4}}\right) L \Theta \\
& \quad-8 \alpha\left(V_{0} A B\left(M-E_{n k}+C_{p s}\right) \alpha+4 k V_{1}\right) \\
& \quad \cdot \frac{s^{5 / 2}}{(1-s)^{4}} \mathbf{L \Theta}
\end{aligned}
$$

Thus, we need to find the expectation values of the radial terms $\frac{s^{2}}{(1-s)^{4}}, \frac{s^{2}}{(1-s)^{3}}, \frac{s^{2}}{(1-s)^{2}}, \frac{s^{3 / 2}}{(1-s)^{3}}, \frac{s^{5 / 2}}{(1-s)^{3}}, \frac{s^{3 / 2}}{(1-s)^{4}}, \frac{s^{7 / 2}}{(1-s)^{4}}$ and $\frac{s^{5 / 2}}{(1-s)^{4}}$ taking into account the wave function which we have seen previously in Eqs. (26) and (27):

$$
\begin{aligned}
& \left\langle\frac{s^{2}}{(1-s)^{4}}\right\rangle_{(n l m)}^{s p}=N_{n l}^{\prime 2} \int_{0}^{+\infty}\left\{\frac{s^{2}}{(1-s)^{4}} s^{2 \sqrt{D_{3}^{s}}}\right. \\
& \cdot(1-s)^{1+2 \sqrt{1 / 4+D_{1}^{s}+D_{3}^{s}-D_{2}^{s}}} \\
& \text { · }\left[2 F _ { 1 } \left(-n, n+2 \sqrt{D_{3}^{s}}\right.\right. \\
& +2 \sqrt{1 / 4+D_{1}^{s}+D_{3}^{s}-D_{2}^{s}} \\
& \left.\left.\left.+1 ; 1+2 \sqrt{D_{3}^{s}} ; s\right)\right]^{2}\right\} d r \\
& \left\langle\frac{s^{2}}{(1-s)^{3}}\right\rangle_{(n l m)}^{s p}=N_{n l}^{\prime 2} \int_{0}^{+\infty}\left\{\frac{s^{2}}{(1-s)^{3}} s^{2 \sqrt{D_{3}^{s}}}\right. \\
& \cdot(1-s)^{1+2 \sqrt{1 / 4+D_{1}^{s}+D_{3}^{s}-D_{2}^{s}}} \\
& \text { · }\left[2 F _ { 1 } \left(-n, n+2 \sqrt{D_{3}^{s}}\right.\right. \\
& +2 \sqrt{1 / 4+D_{1}^{s}+D_{3}^{s}-D_{2}^{s}} \\
& \left.\left.\left.+1 ; 1+2 \sqrt{D_{3}^{s}} ; s\right)\right]^{2}\right\} d r \\
& \left\langle\frac{s^{2}}{(1-s)^{2}}\right\rangle_{(n l m)}^{s p}=N_{n l}^{\prime 2} \int_{0}^{+\infty}\left\{\frac{s^{2}}{(1-s)^{2}} s^{2 \sqrt{D_{3}^{s}}}\right. \\
& \cdot(1-s)^{1+2 \sqrt{1 / 4+D_{1}^{s}+D_{3}^{s}-D_{2}^{s}}} \\
& \text { · }\left[2 F _ { 1 } \left(-n, n+2 \sqrt{D_{3}^{s}}\right.\right. \\
& +2 \sqrt{1 / 4+D_{1}^{s}+D_{3}^{s}-D_{2}^{s}} \\
& \left.\left.\left.+1 ; 1+2 \sqrt{D_{3}^{s}} ; s\right)\right]^{2}\right\} d r \\
& \left\langle\frac{s^{3 / 2}}{(1-s)^{3}}\right\rangle_{(n l m)}^{s p}=N_{n l}^{\prime 2} \int_{0}^{+\infty}\left\{\frac{s^{3 / 2}}{(1-s)^{3}} s^{2 \sqrt{D_{3}^{s}}}\right. \\
& \cdot(1-s)^{1+2 \sqrt{1 / 4+D_{1}^{s}+D_{3}^{s}-D_{2}^{s}}} \\
& \text { - }\left[2 F _ { 1 } \left(-n, n+2 \sqrt{D_{3}^{s}}\right.\right. \\
& +2 \sqrt{1 / 4+D_{1}^{s}+D_{3}^{s}-D_{2}^{s}} \\
& \left.\left.\left.+1 ; 1+2 \sqrt{D_{3}^{s}} ; s\right)\right]^{2}\right\} d r \\
& \left\langle\frac{s^{5 / 2}}{(1-s)^{3}}\right\rangle_{(n l m)}^{s p}=N_{n l}^{\prime 2} \int_{0}^{+\infty}\left\{\frac{s^{5 / 2}}{(1-s)^{3}} s^{2 \sqrt{D_{3}^{s}}}\right. \\
& \cdot(1-s)^{1+2 \sqrt{1 / 4+D_{1}^{s}+D_{3}^{s}-D_{2}^{s}}} \\
& \text { · }\left[2 F _ { 1 } \left(-n, n+2 \sqrt{D_{3}^{s}}\right.\right. \\
& +2 \sqrt{1 / 4+D_{1}^{s}+D_{3}^{s}-D_{2}^{s}} \\
& \left.\left.\left.+1 ; 1+2 \sqrt{D_{3}^{s}} ; s\right)\right]^{2}\right\} d r \\
& \left\langle\frac{s^{3 / 2}}{(1-s)^{4}}\right\rangle_{(n l m)}^{s p}=N_{n l}^{\prime 2} \int_{0}^{+\infty}\left\{\frac{s^{3 / 2}}{(1-s)^{4}} s^{2 \sqrt{D_{3}^{s}}}\right. \\
& \cdot(1-s)^{1+2 \sqrt{1 / 4+D_{1}^{s}+D_{3}^{s}-D_{2}^{s}}} \\
& \text { - }\left[2 F _ { 1 } \left(-n, n+2 \sqrt{D_{3}^{s}}\right.\right. \\
& +2 \sqrt{1 / 4+D_{1}^{s}+D_{3}^{s}-D_{2}^{s}} \\
& \left.\left.\left.+1 ; 1+2 \sqrt{D_{3}^{s}} ; s\right)\right]^{2}\right\} d r
\end{aligned}
$$




$$
\begin{aligned}
\left\langle\frac{s^{7 / 2}}{(1-s)^{4}}\right\rangle_{(n l m)}^{s p}= & N_{n l}^{\prime 2} \int_{0}^{+\infty}\left\{\frac{s^{7 / 2}}{(1-s)^{4}} s^{2 \sqrt{D_{3}^{s}}}\right. \\
& \cdot(1-s)^{1+2 \sqrt{1 / 4+D_{1}^{s}+D_{3}^{s}-D_{2}^{s}}} \\
& \cdot\left[2 F _ { 1 } \left(-n, n+2 \sqrt{D_{3}^{s}}\right.\right. \\
& +2 \sqrt{1 / 4+D_{1}^{s}+D_{3}^{s}-D_{2}^{s}} \\
& \left.\left.\left.+1 ; 1+2 \sqrt{D_{3}^{s}} ; s\right)\right]^{2}\right\} d r \\
\left\langle\frac{s^{5 / 2}}{(1-s)^{4}}\right\rangle_{(n l m)}^{s p}= & N_{n l}^{\prime 2} \int_{0}^{+\infty}\left\{\frac{s^{5 / 2}}{(1-s)^{4}} s^{2 \sqrt{D_{3}^{s}}}\right. \\
& \cdot(1-s)^{1+2 \sqrt{1 / 4+D_{1}^{s}+D_{3}^{s}-D_{2}^{s}}} \\
& \cdot\left[2 F _ { 1 } \left(-n, n+2 \sqrt{D_{3}^{s}}\right.\right. \\
& +2 \sqrt{1 / 4+D_{1}^{s}+D_{3}^{s}-D_{2}^{s}} \\
& \left.\left.\left.+1 ; 1+2 \sqrt{D_{3}^{s}} ; s\right)\right]^{2}\right\} d r
\end{aligned}
$$

These relationships can be expressed in terms of a variable $s$ as follows:

$$
\begin{aligned}
\left\langle\frac{s^{2}}{(1-s)^{4}}\right\rangle_{(n l m)}^{s p}= & V_{n l} \int_{0}^{+1}\left\{s^{2 \sqrt{D_{3}^{s}}+1}\right. \\
& \cdot(1-s)^{2 \sqrt{1 / 4+D_{1}^{s}+D_{3}^{s}-D_{2}^{s}}-3} \\
& \cdot\left[2 F _ { 1 } \left(-n, n+2 \sqrt{D_{3}^{s}}\right.\right. \\
& +2 \sqrt{1 / 4+D_{1}^{s}+D_{3}^{s}-D_{2}^{s}} \\
& \left.\left.\left.+1 ; 1+2 \sqrt{D_{3}^{s}} ; s\right)\right]^{2}\right\} d s \\
\left\langle\frac{s^{2}}{(1-s)^{3}}\right\rangle_{(n l m)}^{s p} & V_{n l} \int_{0}^{+1} s^{2 \sqrt{D_{3}^{s}}+1} \\
& \cdot(1-s)^{2 \sqrt{1 / 4+D_{1}^{s}+D_{3}^{s}-D_{2}^{s}}-2} \\
& \cdot\left[2 F _ { 1 } \left(-n, n+2 \sqrt{D_{3}^{s}}\right.\right. \\
& +2 \sqrt{1 / 4+D_{1}^{s}+D_{3}^{s}-D_{2}^{s}} \\
& \left.\left.+1 ; 1+2 \sqrt{D_{3}^{s}} ; s\right)\right]^{2} d s \\
& +1 \\
\left\langle\frac{s^{2}}{(1-s)^{2}}\right\rangle_{(n l m)}^{s p} & V_{0}^{2 \sqrt{D_{3}^{s}}+1} \\
& \cdot(1-s)^{2 \sqrt{1 / 4+D_{1}^{s}+D_{3}^{s}-D_{2}^{s}}-1} \\
& \cdot\left[2 F _ { 1 } \left(-n, n+2 \sqrt{D_{3}^{s}}\right.\right. \\
& +2 \sqrt{1 / 4+D_{1}^{s}+D_{3}^{s}-D_{2}^{s}} \\
& \left.\left.+1 ; 1+2 \sqrt{D_{3}^{s}} ; s\right)\right]^{2} d s
\end{aligned}
$$

$$
\begin{aligned}
& \left\langle\frac{s^{3 / 2}}{(1-s)^{3}}\right\rangle_{(n l m)}^{s p}=V_{n l} \int_{0}^{+1} s^{2 \sqrt{D_{3}^{s}+1 / 2}} \\
& \cdot(1-s)^{2 \sqrt{1 / 4+D_{1}^{s}+D_{3}^{s}-D_{2}^{s}-2}} \\
& \text { - }\left[2 F _ { 1 } \left(-n, n+2 \sqrt{D_{3}^{s}}\right.\right. \\
& +2 \sqrt{1 / 4+D_{1}^{s}+D_{3}^{s}-D_{2}^{s}} \\
& \left.\left.+1 ; 1+2 \sqrt{D_{3}^{s}} ; s\right)\right]^{2} d s \\
& \left\langle\frac{s^{5 / 2}}{(1-s)^{3}}\right\rangle_{(n l m)}^{s p}=V_{n l} \int_{0}^{+1} s^{2 \sqrt{D_{3}^{s}+3 / 2}} \\
& \cdot(1-s)^{2 \sqrt{1 / 4+D_{1}^{s}+D_{3}^{s}-D_{2}^{s}-2}} \\
& \text { · }\left[2 F _ { 1 } \left(-n, n+2 \sqrt{D_{3}^{s}}\right.\right. \\
& +2 \sqrt{1 / 4+D_{1}^{s}+D_{3}^{s}-D_{2}^{s}} \\
& \left.\left.+1 ; 1+2 \sqrt{D_{3}^{s}} ; s\right)\right]^{2} d s \\
& \left\langle\frac{s^{3 / 2}}{(1-s)^{4}}\right\rangle_{(n l m)}^{s p}=V_{n l} \int_{0}^{+1} s^{2 \sqrt{D_{3}^{s}}+1 / 2} \\
& \cdot(1-s)^{2 \sqrt{1 / 4+D_{1}^{s}+D_{3}^{s}-D_{2}^{s}}-3} \\
& \text { · }\left[2 F _ { 1 } \left(-n, n+2 \sqrt{D_{3}^{s}}\right.\right. \\
& +2 \sqrt{1 / 4+D_{1}^{s}+D_{3}^{s}-D_{2}^{s}} \\
& \left.\left.+1 ; 1+2 \sqrt{D_{3}^{s}} ; s\right)\right]^{2} d s \\
& \left\langle\frac{s^{7 / 2}}{(1-s)^{4}}\right\rangle_{(n l m)}^{s p}=V_{n l} \int_{0}^{+1} s^{2 \sqrt{D_{3}^{s}}+5 / 2} \\
& \cdot(1-s)^{2 \sqrt{1 / 4+D_{1}^{s}+D_{3}^{s}-D_{2}^{s}-3}} \\
& \text { - }\left[2 F _ { 1 } \left(-n, n+2 \sqrt{D_{3}^{s}}\right.\right. \\
& +2 \sqrt{1 / 4+D_{1}^{s}+D_{3}^{s}-D_{2}^{s}} \\
& \left.\left.+1 ; 1+2 \sqrt{D_{3}^{s}} ; s\right)\right]^{2} d s \\
& \left\langle\frac{s^{5 / 2}}{(1-s)^{4}}\right\rangle_{(n l m)}^{s p}=V_{n l} \int_{0}^{+1} s^{2 \sqrt{D_{3}^{s}+3 / 2}} \\
& \cdot(1-s)^{2 \sqrt{1 / 4+D_{1}^{s}+D_{3}^{s}-D_{2}^{s}}-3} \\
& \text { - }\left[2 F _ { 1 } \left(-n, n+2 \sqrt{D_{3}^{s}}\right.\right. \\
& +2 \sqrt{1 / 4+D_{1}^{s}+D_{3}^{s}-D_{2}^{s}} \\
& \left.\left.+1 ; 1+2 \sqrt{D_{3}^{s}} ; s\right)\right]^{2} d s
\end{aligned}
$$

Here $V_{n l}=\frac{N_{n l}^{\prime 2}}{2 \alpha}$. With the help of the special integral formula in Eq. (49), we obtain:

$$
\begin{aligned}
\left\langle\frac{s^{2}}{(1-s)^{4}}\right\rangle_{(n l m)}^{s p} & \\
= & V_{n l} \frac{\Gamma\left(2 \sqrt{D_{3}^{s}}+2\right) \Gamma\left(D^{1 s}-2\right)}{\Gamma\left(D^{2 s}\right)} \\
& \cdot{ }_{3} F_{2}\left(-n, D^{n}, D^{1 s}-2 ;\right. \\
& \left.1+2 \sqrt{D_{3}^{s}}, D^{2 s} ; 1\right)
\end{aligned}
$$




$$
\begin{aligned}
& \left\langle\frac{s^{2}}{(1-s)^{3}}\right\rangle_{(n l m)}^{s p} \\
& =V_{n l} \frac{\Gamma\left(2 \sqrt{D_{3}^{s}}+2\right) \Gamma\left(D^{1 s}-1\right)}{\Gamma\left(D^{s}+1\right)} \\
& { }_{3} F_{2}\left(-n, D^{n}, D^{1 s}-1\right. \text {; } \\
& \left.1+2 \sqrt{D_{3}^{s}}, D^{2 s}+1 ; 1\right) \\
& \left\langle\frac{s^{2}}{(1-s)^{2}}\right\rangle_{(n l m)}^{s p} \\
& =V_{n l} \frac{\Gamma\left(2 \sqrt{D_{3}^{s}}+2\right) \Gamma\left(D^{1 s}\right)}{\Gamma\left(D^{2 s}+2\right)} \\
& { }_{3} F_{2}\left(-n, n+D^{2 s}+1, D^{1 s} ;\right. \\
& \left.1+2 \sqrt{D_{3}^{s}}, D^{2 s}+2 ; 1\right) \\
& \left\langle\frac{s^{3 / 2}}{(1-s)^{3}}\right\rangle_{(n l m)}^{s p} \\
& =V_{n l} \frac{\Gamma\left(2 \sqrt{D_{3}^{s}}+3 / 2\right) \Gamma\left(D^{1 s}-1\right)}{\Gamma\left(D^{2 s}+1 / 2\right)} \\
& { }_{3} F_{2}\left(-n, D^{n}, D^{1 s}-1\right. \text {; } \\
& \left.1+2 \sqrt{D_{3}^{s}}, D^{2 s}+1 / 2 ; 1\right) \\
& \left\langle\frac{s^{5 / 2}}{(1-s)^{3}}\right\rangle_{(n l m)}^{s p} \\
& =V_{n l} \frac{\Gamma\left(2 \sqrt{D_{3}^{s}}+5 / 2\right) \Gamma\left(D^{1 s}-1\right)}{\Gamma\left(D^{2 s}+3 / 2\right)} \\
& { }_{3} F_{2}\left(-n, D^{n}, D^{1 s}-1\right. \text {; } \\
& \left.1+2 \sqrt{D_{3}^{s}}, D^{2 s}+3 / 22 ; 1\right) \\
& \left\langle\frac{s^{3 / 2}}{(1-s)^{4}}\right\rangle_{(n l m)}^{s p} \\
& =\frac{V_{n l} \Gamma\left(2 \sqrt{D_{3}^{s}}+3 / 2\right) \Gamma\left(D^{1 s}-2\right)}{\Gamma\left(D^{2 s}-1 / 2\right)} \\
& \cdot{ }_{3} F_{2}\left(-n, D^{n}, D^{1 s}-2\right. \text {; } \\
& \left.1+2 \sqrt{D_{3}^{s}}, D^{2 s}-1 / 2 ; 1\right) \\
& \left\langle\frac{s^{7 / 2}}{(1-s)^{4}}\right\rangle_{(n l m)}^{s p} \\
& =\frac{V_{n l} \Gamma\left(2 \sqrt{D_{3}^{s}}+7 / 2\right) \Gamma\left(D^{1 s}-2\right)}{\Gamma\left(D^{2 s}+3 / 2\right)} \\
& \cdot{ }_{3} F_{2}\left(-n, D^{n}, D^{1 s}-2\right. \text {; } \\
& \left.1+2 \sqrt{D_{3}^{s}}, D^{2 s}+3 / 2 ; 1\right) \\
& \left\langle\frac{s^{5 / 2}}{(1-s)^{4}}\right\rangle_{(n l m)}^{s p} \\
& =\frac{V_{n l} \Gamma\left(2 \sqrt{D_{3}^{s}}+5 / 2\right) \Gamma\left(D^{1 s}-2\right)}{\Gamma\left(D^{2 s}+1 / 2\right)} \\
& { }_{3} F_{2}\left(-n, D^{n}, D^{1 s}-2\right. \text {; } \\
& \left.1+2 \sqrt{D_{3}^{s}}, D^{2 s}+1 / 2 ; 1\right)
\end{aligned}
$$

with $\quad D^{n}=n+D^{2 s}+1, \quad D^{1 s}=2 \sqrt{1 / 4+D_{1}^{s}+D_{3}^{s}-D_{2}^{s}}$ and $D^{2 s}=2 \sqrt{D_{3}^{s}}+2 \sqrt{1 / 4+D_{1}^{s}+D_{3}^{s}-D_{2}^{s}}$. In addition to the other eight expectations values corresponding to the pseudospin symmetry.

$$
\begin{aligned}
& \left\langle\frac{s^{2}}{(1-s)^{4}}\right\rangle_{(n l m)}^{p s p} \\
& =\frac{V_{n l} \Gamma\left(2 \sqrt{Q_{3}^{s}}+2\right) \Gamma\left(Q^{1 s}-2\right)}{\Gamma\left(Q^{2 s}\right)} \\
& \cdot{ }_{3} F_{2}\left(-n, Q^{n} 1, Q^{1 s}-2\right. \text {; } \\
& \left.1+2 \sqrt{Q_{3}^{s}}, Q^{2 s} ; 1\right) \\
& \left\langle\frac{s^{2}}{(1-s)^{3}}\right\rangle_{(n l m)}^{p s p} \\
& =\frac{V_{n l} \Gamma\left(2 \sqrt{Q_{3}^{p s}}+2\right) \Gamma\left(Q^{1 p s}-1\right)}{\Gamma\left(Q^{p s}+1\right)} \\
& \cdot{ }_{3} F_{2}\left(-n, Q^{n}, Q^{1 p s}-1\right. \text {; } \\
& \left.1+2 \sqrt{Q_{3}^{s}}, Q^{2 p s}+1 ; 1\right) \\
& \left\langle\frac{s^{2}}{(1-s)^{2}}\right\rangle_{(n l m)}^{p s p} \\
& =\frac{V_{n l} \Gamma\left(2 \sqrt{Q p_{3}^{s}}+2\right) \Gamma\left(Q^{1 p s}\right)}{\Gamma\left(Q^{2 p s}+2\right)} \\
& { }_{3} F_{2}\left(-n, Q^{n}, Q^{1 p s}\right. \text {; } \\
& \left.1+2 \sqrt{Q p_{3}^{s}}, Q^{2 p s}+2 ; 1\right) \\
& \left\langle\frac{s^{3 / 2}}{(1-s)^{3}}\right\rangle_{(n l m)}^{p s p} \\
& =\frac{V_{n l} \Gamma\left(2 \sqrt{Q_{3}^{p s}}+3 / 2\right) \Gamma\left(Q^{1 p s}-1\right)}{\Gamma\left(Q^{2 p s}+1 / 2\right)} \\
& \cdot{ }_{3} F_{2}\left(-n, Q^{n}, Q^{1 p s}-1\right. \text {; } \\
& \left.1+2 \sqrt{Q_{3}^{p s}}, Q^{2 p s}+1 / 2 ; 1\right) \\
& \left\langle\frac{s^{5 / 2}}{(1-s)^{3}}\right\rangle_{(n l m)}^{p s p} \\
& =\frac{V_{n l} \Gamma\left(2 \sqrt{Q_{3}^{p s}}+5 / 2\right) \Gamma\left(Q^{1 p s}-1\right)}{\Gamma\left(Q^{2 p s}+3 / 2\right)} \\
& { }_{3} F_{2}\left(-n, Q^{n}, Q^{1 p s}-1\right. \text {; } \\
& \left.1+2 \sqrt{Q p_{3}^{s}}, Q^{2 p s}+3 / 2 ; 1\right) \\
& \left\langle\frac{s^{3 / 2}}{(1-s)^{4}}\right\rangle_{(n l m)}^{p s p} \\
& =\frac{V_{n l} \Gamma\left(2 \sqrt{Q_{3}^{p s}}+3 / 2\right) \Gamma\left(Q^{1 p s}-2\right)}{\Gamma\left(Q^{2 s}-1 / 2\right)} \\
& { }_{3} F_{2}\left(-n, Q^{n}, Q^{1 p s}-2\right. \text {; } \\
& \left.1+2 \sqrt{Q p_{3}^{s}}, Q^{2 p s}-1 / 2 ; 1\right) \\
& \left\langle\frac{s^{7 / 2}}{(1-s)^{4}}\right\rangle_{(n l m)}^{p s p} \\
& =\frac{V_{n l} \Gamma\left(2 \sqrt{Q_{3}^{p s}}+7 / 2\right) \Gamma\left(Q^{1 p s}-2\right)}{\Gamma\left(Q^{2 p s}+3 / 2\right)} \\
& \cdot{ }_{3} F_{2}\left(-n, Q^{n}, Q^{1 p s}-2\right. \text {; } \\
& \left.1+2 \sqrt{Q_{3}^{p s}}, Q^{2 p s}+3 / 2 ; 1\right)
\end{aligned}
$$




$$
\begin{aligned}
\left\langle\frac{s^{5 / 2}}{(1-s)^{4}}\right\rangle_{(n l m)}^{p s p} & V_{n l} \Gamma\left(2 \sqrt{Q_{3}^{p s}}+5 / 2\right) \Gamma\left(Q^{1 p s}-2\right) \\
= & \frac{\Gamma\left(Q^{2 p s}+1 / 2\right)}{\cdot{ }_{3} F_{2}\left(-n, Q^{n}, Q^{1 p s}-2 ;\right.} \\
& \left.1+2 \sqrt{Q_{3}^{p s}}, Q^{2 p s}+1 / 2 ; 1\right)
\end{aligned}
$$

with $Q^{n}=n+Q^{2 p s}+1, Q^{1 p s}=2 \sqrt{1 / 4+Q_{1}^{p s}+Q_{3}^{p s}-Q_{2}^{p s}}$ and $Q^{2 p s}=2 \sqrt{Q_{3}^{p s}}+2 \sqrt{1 / 4+Q_{1}^{p s}+Q_{3}^{p s}-Q_{2}^{p s}}$. By applying the same philosophical method that we saw in the previous section related to the deformed Klein-Gordon theory, taking into account statistical differences between this theory and deformed Dirac theory. The global relativistic expectation values $\langle\Xi\rangle_{(n l m)}^{s}\left(n, \alpha, A, B, V_{0}, V_{1}\right)$ and $\langle\Xi\rangle_{(n l m)}^{p s}(n, \alpha$, $\left.A, B, V_{0}, V_{1}\right)$ are determined from the following relation:

$$
\begin{aligned}
\langle\Xi\rangle_{(n l m)}^{s}\left(n, \alpha, A, B, V_{0}, V_{1}\right) & 16 \alpha^{4} k(k+1)\left\langle\frac{s^{2}}{(1-s)^{4}}\right\rangle_{(n l m)}^{s p} \\
& -2 k V_{1} \alpha^{2}\left\langle\frac{s^{2}}{(1-s)^{3}}\right\rangle_{(n l m)}^{s p} \\
& +16 V_{1}^{2} \alpha^{4}\left\langle\frac{s^{2}}{(1-s)^{2}}\right\rangle_{(n l m)}^{s p} \\
& +\left(4\left(M+E_{n k}^{s}-C_{s}\right) \alpha^{2} V_{0} B A+2 V_{1}^{2} \alpha^{2}\right) \\
& \cdot\left\langle\frac{s^{3 / 2}}{(1-s)^{3}}\right\rangle_{(n l m)}^{s p}+4\left(M+E_{n k}^{s}-C_{s}\right) \\
& \cdot \alpha^{2}\left(V_{0} B^{2}\left\langle\frac{s^{5 / 2}}{(1-s)^{3}}\right\rangle_{(n l m)}^{s p}\right. \\
& -V_{0} A^{2}\left\langle\frac{s^{3 / 2}}{(1-s)^{4}}\right\rangle_{(n l m)}^{s p} \\
& \left.-V_{0} B^{2}\left\langle\frac{s^{7 / 2}}{(1-s)^{4}}\right\rangle_{(n l m)}^{s p}\right) \\
& -8 \alpha\left(V_{0} A B\left(M+E_{n k}^{s}-C_{s}\right) \alpha+4 k V_{1}\right) \\
& \cdot\left\langle\frac{s^{5 / 2}}{(1-s)^{4}}{ }_{(n l m)}^{s p}\right.
\end{aligned}
$$

and

$$
\begin{aligned}
\langle\Xi\rangle_{(n l m)}^{p s}\left(n, \alpha, A, B, V_{0}, V_{1}\right) & 16 \alpha^{4} k(k-1)\left\langle\frac{s^{2}}{(1-s)^{4}}\right\rangle_{(n l m)}^{p s p} \\
& -2 k V_{1} \alpha^{2}\left\langle\frac{s^{2}}{(1-s)^{3}}\right\rangle_{(n l m)}^{p s p} \\
& +16 V_{1}^{2} \alpha^{4}\left\langle\frac{s^{2}}{(1-s)^{2}}\right\rangle_{(n l m)}^{p s p} \\
& +\left(4\left(M+E_{n k}^{p s}-C_{s}\right) \alpha^{2} V_{0} B A+2 V_{1}^{2} \alpha^{2}\right) \\
& \cdot\left\langle\frac{s^{\frac{3}{2}}}{(1-s)^{3}}\right\rangle_{(n l m)}^{p s p} 4\left(M-E_{n k}^{p s}+C_{p s}\right) \\
& \cdot \alpha^{2}\left(V_{0} B^{2}\left\langle\frac{s^{\frac{5}{2}}}{(1-s)^{3}}\right\rangle_{(n l m)}^{p s p}\right. \\
& -V_{0} A^{2}\left\langle\frac{s^{\frac{3}{2}}}{(1-s)^{4}}\right\rangle_{(n l m)}^{p s p} \\
& \left.-V_{0} B^{2}\left\langle\frac{s^{\frac{7}{2}}}{(1-s)^{4}}\right\rangle_{(n l m)}^{p s p}\right) \\
& -8 \alpha\left(V_{0} A B\left(M-E_{n k}^{p s}+C_{p s}\right) \alpha+4 k V_{1}\right) \\
& \cdot\left\langle\frac{s^{5 / 2}}{\left.(1-s)^{4}\right\rangle_{(n l m)}^{p s p}}\right.
\end{aligned}
$$

We have seen that in the previous section that the eigenvalues of the operator $G^{2}=J^{2}-L^{2}-S^{2}$ are equal to the values $\quad f(j, l, s)=[j(j+1)-l(l+1)-3 / 4] / 2 \quad$ and $f(j, \tilde{l}, \tilde{s})=[j(j+1)-\tilde{l}(\tilde{l}+1)-3 / 4] / 2$ thus, for the case of spin-1/2, the possible values of $j$ are $l \pm 1 / 2$ and $\tilde{l} \pm 1 / 2$ for spin symmetry $f(j, l, s)$ and pseudospin symmetry $f(j, \tilde{l}, \tilde{s})$, which allows us to get and as follows:

$$
\begin{gathered}
f(j, l, s)=\left\{\begin{array}{cc}
\frac{l}{2} & \text { for } j=l+1 / 2 \\
-\frac{l+1}{2} & \text { for } j=l-1 / 2
\end{array}\right. \\
f(j, \tilde{l}, \tilde{s})=\left\{\begin{array}{cc}
\frac{l}{2} & \text { for } j=\tilde{l}+1 / 2 \\
-\frac{l+1}{2} & \text { for } j=\tilde{l}-1 / 2
\end{array}\right.
\end{gathered}
$$

We can express to new relativistic energy

$$
\begin{gathered}
E_{r-n c}^{s p}\left(n, \alpha, A, B, V_{0}, V_{1}, \Theta, \sigma, \chi, j, l, s, m\right) \text { and } \\
E_{r-n c}^{p s}\left(n, \alpha, A, B, V_{0}, V_{1}, \Theta, \sigma, \chi, j, \tilde{l}, \tilde{s}, \widetilde{m}\right)
\end{gathered}
$$

for the massive particle with spin-1/2 interacting with the improved modified Mobius squared potential, in the symmetries of the deformed Dirac symmetries, corresponding to the generalized $n^{\text {th }}$ excited states as follows:

$$
\begin{array}{r}
E_{r-n c}^{s p}\left(n, \alpha, A, B, V_{0}, V_{1}, \Theta, \sigma, \chi, j, l, s, m\right) \\
=E_{n k}^{s}+\langle\Xi\rangle_{(n l m)}^{s}\left(n, \alpha, A, B, V_{0}, V_{1}\right) \\
\left\{\begin{array}{cc}
\frac{l}{2} & \text { for } l+1 / 2 \\
-\frac{l+1}{2} & \text { for } l-1 / 2
\end{array}\right.
\end{array}
$$

and

$$
\begin{gathered}
E_{r-n c}^{p s}\left(n, \alpha, A, B, V_{0}, V_{1}, \Theta, \sigma, \chi, j, \tilde{l}, \tilde{s}, \widetilde{m}\right) \\
=E_{n k}^{p s}+\langle\Xi\rangle_{(n l m)}^{p s}\left(n, \alpha, A, B, V_{0}, V_{1}\right) \\
\left\{\begin{array}{cc}
\frac{\tilde{l}}{2} & \text { for } \tilde{l}+1 / 2 \\
-\frac{\tilde{l}+1}{2} & \text { for } \tilde{l}-1 / 2
\end{array}\right.
\end{gathered}
$$

Now, we consider a special case of interest of the improved modified Mobius square potential, known by the improved Deng-Fan potential is given in Eq. (63) in RNCQM symmetries. We apply the steps in Eq. (64) that allow us to 
cross from the improved modified Mobius square potential to our case to find without returning the previous calculations and let's start with the global relativistic expectation values $\langle\Xi\rangle_{(n l m)}^{s-d f}\left(n, \alpha, A, B, V_{0}, V_{1}\right)$ and $\langle\Xi\rangle_{(n l m)}^{p s-d f}\left(n, \alpha, A, B, V_{0}\right.$, $V_{1}$ ) under the improved Deng-Fan potential in the deformed Dirac theory symmetries are determined from the following relation:

$$
\begin{aligned}
\langle\Xi\rangle_{(n l m)}^{s-d f}\left(n, \alpha, A, B, V_{0}, V_{1}\right) & 16 \alpha^{4} k(k+1)\left\langle\frac{s^{2}}{(1-s)^{4}}\right\rangle_{(n l m)}^{s p} \\
& -2 k V_{1} \alpha^{2}\left\langle\frac{s^{2}}{(1-s)^{3}}\right\rangle_{(n l m)}^{s p} \\
& +16 V_{1}^{2} \alpha^{4}\left\langle\frac{s^{2}}{(1-s)^{2}}\right\rangle_{(n l m)}^{s p} \\
& -\left(4\left(M+E_{n k}^{s-d f}-C_{s}\right) \alpha^{2} D_{e}(1+b)\right. \\
& \left.+2 V_{1}^{2} \alpha^{2}\right)\left\langle\frac{s^{3 / 2}}{(1-s)^{3}}\right\rangle_{(n l m)}^{s p} \\
& +4\left(M+E_{n k}^{s-d f}-C_{s}\right) \alpha^{2} \\
& +\left(D_{e}(1+b)^{2}\left\langle\frac{s^{5 / 2}}{(1-s)^{3}}\right\rangle_{(n l m)}^{s p}\right. \\
& -D_{e}\left\langle\frac{s^{3 / 2}}{(1-s)^{4}}\right\rangle_{(n l m)}^{s p} \\
& -D_{e}(1+b)^{2}\left\langle\frac{s^{7 / 2}}{(1-s)^{4}}\right\rangle_{(n l m)}^{s p} \\
& +8 \alpha\left(D_{e}(1+b)\left(M+E_{n k}^{s-d f}-C_{s}\right) \alpha\right. \\
& \left.-4 k V_{1}\right)\left\langle\frac{s^{5 / 2}}{(1-s)^{4}}\right\rangle_{(n l m)}^{s p}
\end{aligned}
$$

and

$$
\begin{aligned}
\langle\Xi\rangle_{(n l m)}^{p s-d f}\left(n, \alpha, A, B, V_{0}, V_{1}\right) & 16 \alpha^{4} k(k-1)\left\langle\frac{s^{2}}{(1-s)^{4}}\right\rangle_{(n l m)}^{p s p} \\
& -2 k V_{1} \alpha^{2}\left\langle\frac{s^{2}}{(1-s)^{3}}\right\rangle_{(n l m)}^{p s p} \\
& +16 V_{1}^{2} \alpha^{4}\left\langle\frac{s^{2}}{(1-s)^{2}}\right\rangle_{(n l m)}^{p s p} \\
& -\left(4\left(M+E_{n k}^{p s-d f}-C_{s}\right) \alpha^{2} D_{e}(1+b)\right. \\
& \left.+2 V_{1}^{2} \alpha^{2}\right)\left\langle\frac{s^{3 / 2}}{(1-s)^{3}}\right\rangle_{(n l m)}^{p s p} \\
& +4\left(M+E_{n k}^{p s-d f}-C_{p s}\right) \alpha^{2} \\
& +\left(D_{e}(1+b)^{2}\left\langle\frac{s^{5 / 2}}{(1-s)^{3}}\right\rangle_{(n l m)}^{p s p}\right. \\
& -D_{e}\left\langle\frac{s^{3 / 2}}{(1-s)^{4}}\right\rangle_{(n l m)}^{p s p} \\
& -D_{e}(1+b)^{2}\left\langle\frac{s^{7 / 2}}{(1-s)^{4}}\right\rangle_{(n l m)}^{p s p} \\
& +8 \alpha\left(D_{e}(1+b)\left(M+E_{n k}^{p s-d f}-C_{p s}\right) \alpha\right. \\
& \left.-4 k V_{1}\right)\left\langle\frac{s^{5 / 2}}{\left.(1-s)^{4}\right\rangle_{(n l m)}^{p s p}}\right.
\end{aligned}
$$

The new relativistic energy for the fermionic massive spin-1/2 particles interacting with the improved Deng-Fan potential model in the deformed Dirac theory symmetries $E_{r-n c}^{s p-d f}\left(n, \alpha, A, B, V_{0}, V_{1}, \Theta, \sigma, \chi, j, l, s, m\right)$ and $E_{r-n c}^{p s-d f}(n, \alpha, A, B$, $\left.V_{0}, V_{1}, \Theta, \sigma, \chi, j, \tilde{l}, \tilde{s}, \widetilde{m}\right)$, corresponding to the generalized $n^{t h}$ excited states as follows:

$$
\begin{array}{r}
E_{r-n c}^{s p-d f}\left(n, \alpha, A, B, V_{0}, V_{1}, \Theta, \sigma, \chi, j, l, s, m\right) \\
=E_{n k}^{s-d f}+\langle\Xi\rangle_{(n l m)}^{s-d f}\left(n, \alpha, A, B, V_{0}, V_{1}\right) \\
\left\{\begin{array}{cc}
\frac{l}{2} & \text { for } l+1 / 2 \\
-\frac{l+1}{2} & \text { for } l-1 / 2
\end{array}\right.
\end{array}
$$

and

$$
\begin{gathered}
E_{r-n c}^{p s-d f}\left(n, \alpha, A, B, V_{0}, V_{1}, \Theta, \sigma, \chi, j, \tilde{l}, \tilde{s}, \tilde{m}\right) \\
=E_{n k}^{p s-d f}+\langle\Xi\rangle_{(n l m)}^{p s-d f}\left(n, \alpha, A, B, V_{0}, V_{1}\right) \\
\left\{\begin{array}{cc}
\frac{\tilde{l}}{2} & \text { for } \tilde{l}+1 / 2 \\
-\frac{\tilde{l}+1}{2} & \text { for } \tilde{l}-1 / 2
\end{array}\right.
\end{gathered}
$$

Here $E_{n k}^{s-d f}$ and $E_{n k}^{p s-d f}$ are the relativistic energy spectra for the Deng-Fan potential in the ordinary Dirac theory which is determined for $H=0$ and $V_{1}=0.2,78$

\section{NONRELATIVISTIC STUDY OF IMSPM}

\subsection{NONRELATIVISTIC SPECTRUM UNDER THE IMSPM}

In this section, we want to derive the nonrelativistic spectrum, which is produced by the effect of an improved modified Mobius squared potential model for diatomic molecules such as $\mathrm{H}_{2}, \mathrm{I}_{2}$, CO, NO, and $\mathrm{HCl}$. From Eqs. (1) and (11), we can write this potential in the nonrelativistic noncommutative three-dimensional real space NRNCQM symmetries as follows:

$$
V_{n c}^{m s p}(r)=-V_{0}\left(\frac{A+B e^{-2 \alpha r}}{1-e^{-2 \alpha r}}\right)^{2}+V_{n r-p e r t}^{m s p}(r)
$$

Where $V_{p e r t}^{m s p}(r)$ is the perturbative potential in nonrelativistic noncommutative three-dimensional real space NRNCQM symmetries:

$$
\begin{aligned}
V_{n r-p e r t}^{m s p}(r)= & \frac{l(l+1)}{r^{4}} \mathbf{L} \Theta-\frac{\partial V_{n c}^{m s p}(r)}{\partial r} \frac{\mathbf{L} \Theta}{2 r} \\
& +O\left(\Theta^{2}\right)
\end{aligned}
$$

The first term due to the centrifuge term $\frac{l(l+1)}{r_{n c}^{2}}$ in NRNCQM (Eq. (39)) which equals the usual centrifuge term $\frac{l(l+1)}{r^{2}}$ plus the perturbative centrifuge term $\frac{l(l+1)}{r^{4}} \mathbf{L} \Theta$ while the second term in Eq. (119) is produced with the effect of modified Mobius squared potential. We have applied the approximation type suggested by (Greene and Aldrich) for a shortrange potential that is an excellent approximation to the centrifugal term for Eckart potential and we calculate $\frac{\partial V_{n c}^{m s p}(r)}{\partial r}$ (see Eq. (43)). Now, substituting Eq. (43) into Eq. (103) and we apply the approximation in Eq. (45), we get the perturbative potential created with the effect of the improved modified Mobius squared potential model in NRNCQM symmetries as follows: 


$$
\begin{aligned}
V_{n r-p e r t}^{m s p}(r)= & \frac{16 l(l+1) \alpha^{4} s^{2}}{(1-s)^{4}} \\
& -4 \alpha^{2}\left(\frac{V_{0} A B s^{3 / 2}}{(1-s)^{3}}+\frac{V_{0} B^{2} s^{5 / 2}}{(1-s)^{3}}\right. \\
& -\frac{V_{0} A^{2} s^{3 / 2}}{(1-s)^{4}}-\frac{V_{0} B^{2} s^{7 / 2}}{(1-s)^{4}} \\
& \left.\left.-\frac{2 V_{0} A B s^{5 / 2}}{(1-s)^{4}}\right)\right) \mathbf{L \Theta}+O\left(\Theta^{2}\right)
\end{aligned}
$$

Thus, we need the know the expectation values of $\frac{s^{2}}{(1-s)^{4}}$, $\frac{s^{3 / 2}}{(1-s)^{3}}, \frac{s^{5 / 2}}{(1-s)^{3}}, \frac{s^{3 / 2}}{(1-s)^{4}}, \frac{s^{7 / 2}}{(1-s)^{4}}$ and $\frac{s^{5 / 2}}{(1-s)^{4}}$ to find the nonrelativistic energy corrections produced by the perturbative potential $V_{n r-p e r t}^{m s p}(s)$. By using the expectations values obtained in Eqs. (50.1), (50.2, (50.3), (50.4), (50.5), and (50.6) for the $n^{\text {th }}$ excited state, we get the corresponding global expectation values $\langle\Xi\rangle_{(n l m)}^{N R m s p}\left(n, \alpha, A, B, V_{0}\right)$ as follows:

$$
\begin{aligned}
\langle\Xi\rangle_{(n l m)}^{N R m s p}\left(n, \alpha, A, B, V_{0}\right) & 16 l(l+1) \alpha^{4}\left\langle\frac{s^{2}}{(1-s)^{4}}\right\rangle_{(n l m)} \\
& -4 \alpha^{2}\left(V_{0} A B\left\langle\frac{s^{3 / 2}}{(1-s)^{3}}\right\rangle_{(n l m)}\right. \\
& +V_{0} B^{2}\left\langle\frac{s^{5 / 2}}{(1-s)^{3}}\right\rangle_{(n l m)} \\
& -V_{0} A^{2}\left\langle\frac{s^{3 / 2}}{(1-s)^{4}}\right\rangle_{(n l m)} \\
& -V_{0} B^{2}\left\langle\frac{s^{7 / 2}}{(1-s)^{4}}\right\rangle_{(n l m)} \\
& \left.-2 V_{0} A B\left\langle\frac{s^{5 / 2}}{(1-s)^{4}}\right\rangle_{(n l m)}\right)
\end{aligned}
$$

By following the same physical methodology that we devoted in our relativistic previous study, the energy corrections $\Delta E_{m s p}^{n r}\left(n, \alpha, A, B, V_{0}, \Theta, \sigma, \chi, j, l, s, m\right)$ for the $n^{t h}$ excited-state due to the spin-orbit coupling, modified Zeeman effect, and nonrelativistic perturbed Fermi gas potential which was induced under the influence of the improved modified Mobius squared potential model in NRNCQM symmetries as follows:

$$
\begin{aligned}
\Delta & E_{m s p}^{n r}\left(n, \alpha, A, B, V_{0}, \Theta, \sigma, \chi, j, l, s, m\right) \\
= & \langle\Xi\rangle_{(n l m)}^{N R m s p}\left(n, \alpha, A, B, V_{0}\right)(\Theta f(j, l, s) \\
& +\sigma \aleph+m \chi \Omega m)
\end{aligned}
$$

As a direct consequence, the new nonrelativistic energy $E_{n r-n c}^{m s p}\left(n, \alpha, A, B, V_{0}, \Theta, \sigma, \chi, j, l, s, m\right)$ produced with the improved modified Mobius squared potential model, in the symmetries of NRNCQM for the $n^{\text {th }}$ generalized excited states, the sum of the energy corrections $\Delta E_{m s p}^{n r}\left(n, \alpha, A, B, V_{0}, \Theta, \sigma, \chi, j, l, s, m\right)$ plus the nonrelativistic energy $E_{n l}^{n r}$ produced with the main part of potential (the modified Mobius squared potential) in NRQM as follows:

$$
\begin{gathered}
E_{n r-n c}^{m s p}\left(n, \alpha, A, B, V_{0}, \Theta, \sigma, \chi, j, l, s, m\right) \\
=E_{n l}^{n r}+\langle\Xi\rangle_{(n l m)}^{N R m s p}\left(n, \alpha, A, B, V_{0}\right) \\
\cdot(\Theta f(j, l, s)+\sigma \aleph m+\chi \Omega m)
\end{gathered}
$$

The nonrelativistic energy $E_{n l}^{n r}$ due to the effect of the modified Mobius squared potential in Eq. (31).

\subsection{STUDY OF COMPOSITE SYSTEMS}

Now, considering composite systems such as molecules made of $N=2$ particles of masses $m_{n}(n=1,2)$ in the frame of noncommutative algebra, it is worth taking into account features of descriptions of the systems in space. In NRQM symmetries, it was obtained those composite systems with different masses are described with different noncommutative parameters ${ }^{78-81}$ :

$$
\begin{aligned}
& {\left[x_{n c \mu}^{(S, H, I)_{*}}, x_{n c \nu}{ }^{(S, H, I)}\right]=i \theta_{\mu \nu}^{c}} \\
& \text { with } \theta_{\mu \nu}^{c}=\sum_{n=1}^{2} \mu_{n}^{2} \theta_{\mu \nu}^{(n)}
\end{aligned}
$$

with $\mu_{n}=\frac{m_{n}}{\sum_{n} m_{n}}$, the indices $(n=1,2)$ label the particle, and $\theta_{\mu \nu}^{(n)}$ is the parameter of noncommutativity, corresponding to the particle of mass $m_{n}$. Note that in the case of a system of two particles with the same mass $m_{1}=m_{2}$ such as the homogeneous $\left(\mathrm{H}_{2}\right.$ and $\left.\mathrm{I}_{2}\right)$ diatomic molecules the parameter $\theta_{\mu \nu}^{(n)}=\theta_{\mu v}$. Thus, the two parameters $\Theta$ and $\sigma$ which appears in Eq. (107) are changed to the new form:

$$
\begin{aligned}
\Lambda^{c 2}= & \left(\sum_{n=1}^{2} \mu_{n}^{2} \Lambda_{12}^{(n)}\right)^{2}+\left(\sum_{n=1}^{2} \mu_{n}^{2} \Lambda_{23}^{(n)}\right)^{2} \\
& +\left(\sum_{n=1}^{2} \mu_{n}^{2} \Lambda_{13}^{(n)}\right)^{2}
\end{aligned}
$$

Where $\Lambda^{c}$ can take the parameters $\left(\Theta^{c}, \sigma^{c}\right.$ and $\left.\chi^{c}\right)$. As is mentioned above, in the case of a system of two particles with the same mass $m_{1}=m_{2}$ such as the homogeneous $\left(\mathrm{H}_{2}\right.$ and $\left.\mathrm{I}_{2}\right)$ diatomic molecules $\Theta_{\mu \nu}^{(n)}=\Theta_{\mu \nu}, \sigma_{\mu \nu}^{(n)}=\sigma_{\mu v}$ and $\chi=\chi_{\mu v}$. Finally, we can generalize the nonrelativistic global energy $E_{n r-n c}^{m s p}\left(n, \alpha, A, B, V_{0}, \Theta, \sigma, \chi, j, l, s, m\right)$ under the improved modified Mobius squared potential model considering that composite systems with different masses are described with different noncommutative parameters for the diatomic $(\mathrm{HCl}, \mathrm{CH}, \mathrm{LiH}$, and $\mathrm{CO})$ as:

$$
\begin{gathered}
E_{n r-n c}^{m s p}\left(n, \alpha, A, B, V_{0}, \Theta, \sigma, \chi, j, l, s, m\right) \\
=E_{n l}^{n r}+\langle\Xi\rangle_{(n l m)}^{N R m s p}\left(n, \alpha, A, B, V_{0}\right) \\
\cdot\left(\Theta^{c} f(j, l, s)+\sigma^{c} \aleph m+\chi^{c} \Omega m\right)
\end{gathered}
$$

The ordinary Klien-Gordon equation, as the most wellknown relativistic wave equation describing spin-zero particles, but its extension in DRKGT symmetries under the improved modified Mobius squared potential has a physical behavior similar to the Duffin-Kemmer equation for a meson with spin-s, it can describe a dynamic state of a particle with spin one in the symmetries of relativistic noncommutative quantum mechanics. On the other hand, we have seen the appearance of the spin in the deformed Schrödinger equation, which means that it rises in its physical description of the state of the Dirac equation known in the literature. This is one of the most important new results of this research. Worthwhile it is better to mention that for the two simultaneous limits $(\Theta, \sigma, \chi) \rightarrow(0,0,0)$ and $\left(\Theta^{c}, \sigma^{c}, \chi^{c}\right) \rightarrow(0,0,0)$ we recover the results of the in Okorie et al., ${ }^{1}$ Onyenegecha et al., ${ }^{2}$ and Ikot et al. ${ }^{3}$ 


\section{SUMMARY AND CONCLUSIONS}

In this work, we have found the approximate bound state solutions of DRKGE and DNRSE using the tool of Bopp's shift and standard perturbation theory methods of the improved modified Mobius squared potential in both relativistic (the case of DKGT and DDT symmetries) and nonrelativistic regimes (NRNCQM symmetries) which correspond to high and low energy physics. We have employed the improved approximation scheme to deal with the centrifugal term to obtain the new relativistic bound state solutions $E_{r-n c}^{m s p}\left(n, \alpha, A, B, V_{0}, V_{2}, \Theta, \sigma, \chi, j, l, s, m\right)$ (see Eqs. (60), (96) and (97)) corresponding to the generalized $n^{\text {th }}$ excited states that appear as a sum of the total shift energy $\Delta E_{m s p}^{\text {tot }}$ and the relativistic energy $E_{n l}$ of the modified Mobius squared potential. Furthermore, we have obtained the new nonrelativistic global energy $E_{n r-n c}^{m s p}\left(n, \alpha, A, B, V_{0}, \Theta, \sigma, \chi, j, l, s, m\right)$ of some diatomic molecules such as $\left(\mathrm{N}_{2}, \mathrm{I}_{2}, \mathrm{HCl}, \mathrm{CH}, \mathrm{LiH}\right.$, and $\mathrm{CO}$ ) in NRNCQM symmetries as a sum of the nonrelativistic energy (see Eqs. (107) and (112)) and the perturbative corrections $\Delta E_{m s p}^{n r}\left(n, \alpha, A, B, V_{0}, \Theta, \sigma, \chi, j, l, s, m\right)$ (see Eq. (68)). The new relativistic and nonrelativistic energies appear as a function of the discreet atomic quantum numbers $(n, j, l, s, m)$, the potential parameters $\left(\alpha, A, B, V_{0}\right)$ in addition to noncommutativity three parameters $(\Theta, \sigma, \chi)$. This behavior under study is similar to that of a physical system that is affected by three infinitesimal external influences in comparison to the main potential effect (generalized DengFan potential plus the deformed Eckart potential), but in our case, these effects appear automatically as a result of the new deformation of space-space which presented in Eqs. (3) and (4). Moreover, we have applied our results to composite systems such as molecules made of $N=2$ particles of masses $m_{n}(n=1,2)$. We have also dealt with the special case of the Deng-Fan potential in the DKGT and DDT symmetries (see Eqs. (68) and (100) and (101)). We have observed that the DRKGE under the improved modified Mobius squared potential model becomes similar to the Duffin-Kemmer equation for meson with spin-s, it can describe a dynamic state of the particle with spin-s in the symmetries of DKGT. Moreover, concerning the distorted to the distorted Schrödinger equation, it has been shown that it is equivalent in its physical description of the case of the Dirac equation known in the literature. It is worth mentioning that, for all cases, when we apply the two simultaneous limits $(\Theta, \sigma, \chi) \rightarrow(0,0,0)$ and $\left(\Theta^{c}, \sigma^{c}, \chi^{c}\right) \rightarrow(0,0,0)$, the ordinary physical quantities are recovered (we recover the results of the in Okorie et al., ${ }^{1}$ Onyenegecha et al., ${ }^{2}$ and Ikot et $a .^{3}{ }^{3}$ ). Furthermore, our research findings could also be applied in atomic physics, vibrational and rotational spectroscopy, mass spectra, nuclear physics, and other applications. Finally, given the effectiveness of the methods that we followed in achieving our goal in this research, we advise researchers to apply the same methods to delve more deeply, whether in the relativistic and nonrelativistic regimes for others potentials.

\section{ACKNOWLEDGEMENT}

This work was supported by the Research Fund of Laboratory of Physics and Material Chemistry, University of M'sila, and DGRSDT in Algeria with the project number B00LO2UN280120180001. The author wishes to give their sincere gratitude to the referees for their kind comments which improved the manuscript.

Submitted: June 16, $2021+03$, Accepted: September 09, 2021 $+03$ 


\section{REFERENCES}

1. Okorie US, Ikot AN, Onyeaju MC, Chukwuocha EO. Bound state solutions of Schrödinger equation with modified Mobius square potential (MMSP) and its thermodynamic properties. J Mol Model. 2018;24(10). doi:10.1007/s00894-018-3811-8

2. Onyenegecha CP, Opara AI, Njoku IJ, et al. Analytical solutions of D-dimensional Klein-Gordon equation with modified Mobius squared potential. Results in Physics. 2021;25:104144. doi:10.1016/j.rin p.2021.104144

3. Ikot A, Maghsoodi E, Ibanga E, Ituen E, Hassanabadi H. Bound States of the Dirac Equation for Modified Mobius Square Potential Within the Yukawa-Like Tensor Interaction. Proc Natl Acad Sci, India, Sect A Phys Sci. 2016;86(3):433-440. doi:10.100 7/s40010-015-0227-z

4. Ikot AN, Rampho GJ, Amadi PO, Sithole MJ, Okorie US, Lekala MI. Shannon entropy and Fisher information-theoretic measures for Mobius square potential. Eur Phys J Plus. 2020;135(6). doi:10.1140/ep jp/s13360-020-00525-2

5. Ukewuihe UM, Onyenegecha CP, Udensi SC, et al. Mathematics and Computational Sciences. 2021;1(3):1-15.

6. Uduakobong SO, Akpan NI, Edet CO, Rampho JG, Horchani R, Jelassi H. Eur Phys J D. 2021;75(53).

7. Okon IB, Popoola OO, Omugbe E, Antia AD, Isonguyo $\mathrm{CN}$, Ituen $\mathrm{EE}$. Thermodynamic properties and bound state solutions of Schrodinger equation with Mobius square plus screened-Kratzer potential using Nikiforov-Uvarov method. Computational and Theoretical Chemistry. 2020;1196:113132. doi:10.101 6/j.comptc.2020.113132

8. Okorie US, Ikot AN, Ibezim-Ezeani MU, Abdullah HY. Diatomic molecules energy spectra for the generalized Mobius square potential model. Int J Mod Phys B. 2020;34(21):2050209. doi:10.1142/s02179792 20502094

9. Ikot AN, Zarrinkamar S, Ibanga EJ, Maghsoodi E, Hassanabadi H. Pseudospin symmetry of the Dirac equation for a Möbius square plus Mie type potential with a Coulomb-like tensor interaction via SUSYQM. Chinese Phys C. 2014;38(1):013101. doi:10.1088/167 $\underline{4-1137 / 38 / 1 / 013101}$
10. Ikot AN, Okorie US, Sever R, Rampho GJ. Eigensolution, expectation values and thermodynamic properties of the screened Kratzer potential. Eur Phys J Plus. 2019;134(8). doi:10.1140/ep jp/i2019-12783-X

11. Onyenegecha CP, Onate CA, Echendu OK, Ibe AA, Hassanabadi H. Solutions of Schrodinger equation for the modified Mobius square plus Kratzer potential. Eur Phys J Plus. 2020;135(3). doi:10.1140/epjp/s1336 0-020-00304-Z

12. Ikot AN, Maghsoodi E, Zarrinkamar S, Hassanabadi H. Relativistic Spin and Pseudospin Symmetries of Inversely Quadratic Yukawa-like plus Mobius Square Potentials Including a Coulomb-like Tensor Interaction. Few-Body Syst.

2013;54(11):2027-2040. doi:10.1007/s00601-013-070 $\underline{1-6}$

13. Ovando G, Peña JJ, Morales J. On the equivalence of radial potential models for diatomic molecules. Theor Chem Acc. 2016;135(3). doi:10.1007/s00214-01 6-1809-9

14. Doplicher S, Fredenhagen K, Roberts JE. Spacetime quantization induced by classical gravity. Physics Letters B. 1994;331(1-2):39-44. doi:10.1016/03 70-2693(94)90940-7

15. Witten E. Reflections on the Fate of Spacetime. Physics Today. 1996;49(4):24-30. doi:10.1063/1.88149 $\underline{3}$

16. Kempf A, Mangano G, Mann RB. Hilbert space representation of the minimal length uncertainty relation. Phys Rev D. 1995;52(2):1108-1118. doi:10.11 03/physrevd.52.1108

17. Curtright T, Fairlie D, Zachos C. Features of timeindependent Wigner functions. Phys Rev $D$. 1998;58(2):025002. doi:10.1103/physrevd.58.025002

18. Adler RJ, Santiago DI. On gravity and the uncertainty principal. Mod Phys Lett A. 1999;14(20):1371-1381. doi:10.1142/s0217732399001 462

19. Kanazawa T, Lambiase G, Vilasi G, Yoshioka A. Noncommutative Schwarzschild geometry and generalized uncertainty principle. Eur Phys $J$ C. 2019;79(2). doi:10.1140/epjc/s10052-019-6610-1

20. Fabio S. Generalized uncertainty principle in quantum gravity from micro-black hole gedanken experiment. 1999;452(1-2):39-44. 
21. Ho PM, Kao HC. Noncommutative Quantum Mechanics from Noncommutative Quantum Field Theory. Phys Rev Lett. 2002;88(15). doi:10.1103/physr evlett.88.151602

22. Gnatenko KP. Parameters of noncommutativity in Lie-algebraic noncommutative space. Phys Rev $D$. 2019;99(2). doi:10.1103/physrevd.99.026009

23. Bertolami O, Rosa JG, De Aragão CML, Castorina $\mathrm{P}$, Zappalà $\mathrm{D}$. Scaling of variables and the relation between noncommutative parameters in noncommutative quantum mechanics. Mod Phys Lett A. 2006;21(10):795-802. doi:10.1142/s0217732306019 $\underline{840}$

24. Connes A, Douglas MR, Schwarz A. Noncommutative geometry and Matrix theory. J High Energy Phys. 1998;1998(2):003-003. doi:10.1088/112 6-6708/1998/02/003

25. Maireche A. A Model of Modified Klein-Gordon Equation with Modified Scalar-vector Yukawa Potential. Afr Rev Phys. 2020;15(0001):1-11.

26. Zachos C. Geometrical evaluation of star products. Journal of Mathematical Physics. 2000;41(7):5129-5134. doi:10.1063/1.533395

27. Chaichian M, Sheikh-Jabbari MM, Tureanu A. Hydrogen Atom Spectrum and the Lamb Shift in Noncommutative QED. Phys Rev Lett. 2001;86(13):2716-2719. doi:10.1103/physrevlett.86.2 $\underline{716}$

28. Maireche A. Investigations on the Relativistic Interactions in One-Electron Atoms with Modified Yukawa Potential for Spin 1/2 Particles. International Frontier Science Letters. 2017;11:29-44.

29. Zhang J. Fractional angular momentum in noncommutative spaces. Physics Letters $B$. 2004;584(1-2):204-209. doi:10.1016/i.physletb.2004.0 $\underline{1.049}$

30. Maireche A. A New Approach to the Approximate Analytic Solution of the Three-Dimensional Schrödinger Equation for Hydrogenic and Neutral Atoms in the Generalized Hellmann Potential Model. Ukr J Phys. 2020;65(11):987. doi:10.15407/ujpe65.11.9 $\underline{87}$

31. Maireche A. Effects of Two-Dimensional Noncommutative Theories on Bound States Schrödinger Diatomic Molecules under New Modified Kratzer-Type Interactions. International Letters of Chemistry,Physics and Astronomy. 2017;76:1-11.
32. Abreu EMC, Ananias JN, Mendes ACR, Neves C, Oliveira W, Marcial MV. Lagrangian formulation for noncommutative nonlinear systems. Int J Mod Phys A. 2012;27(09):1250053. doi:10.1142/s0217751×1250053 $\underline{4}$

33. Gamboa J, Loewe M, Rojas JC. Noncommutative quantum mechanics. Phys Rev D. 2001;64(6):067901. doi:10.1103/physrevd.64.067901

34. Djemaï AEF, Smail H. On Quantum Mechanics on Noncommutative Quantum Phase Space. Commun Theor Phys (Beijing, China). 2004;41(6):837-844. doi:1 $\underline{0.1088 / 0253-6102 / 41 / 6 / 837}$

35. Yi Y, Kang L, Jian-Hua W, Chi-Yi C. Spin-1/2 relativistic particle in a magnetic field in NC phase space. Chinese Phys C. 2010;34(5):543-547. doi:10.108 8/1674-1137/34/5/005

36. Bertolami O, Leal P. Aspects of phase-space noncommutative quantum mechanics. Physics Letters B. 2015;750:6-11. doi:10.1016/j.physletb.2015.08.024

37. Bertolami O, Rosa JG, de Aragão CML, Castorina P, Zappalà D. Noncommutative gravitational quantum well. Phys Rev D. 2005;72(2):025010-025011. doi:10.1 103/physrevd.72.025010

38. Gamboa J, Loewe M, Rojas JC. Noncommutative quantum mechanics. Phys Rev D. $2001 ; 64(6)$. doi:10.1 103/physrevd.64.067901

39. Li K, Wang J. The topological AC effect on noncommutative phase space. Eur Phys J C.

2007;50(4):1007-1011. doi:10.1140/epjc/s10052-007-0 256-0

40. Wang I, Li K. The HMW effect in noncommutative quantum mechanics. J Phys A: Math Theor. 2007;40(9):2197-2202. doi:10.1088/1751-8113/40/9/0 $\underline{21}$

41. Maireche A. New Bound State Energies for Spherical Quantum Dots in Presence of a Confining Potential Model at Nano and Plank's Scales. NanoWorld J. 2016;1(4):122-129. doi:10.17756/nwj.20 $\underline{16-016}$

42. De Andrade MA, Neves C. Noncommutative mapping from the symplectic formalism. Journal of Mathematical Physics. 2018;59(1):012105. doi:10.106 3/1.4986964

43. Abreu EMC, Neves C, Oliveira W. Noncommutativity from the symplectic point of view. Int J Mod Phys A. 2006;21(26):5359-5369. doi:10.114 $\underline{\text { 2/s0217751x06034094 }}$ 
44. Morse PM. Diatomic Molecules According to the Wave Mechanics. II. Vibrational Levels. Phys Rev. 1929;34(1):57-64. doi:10.1103/physrev.34.57

45. Dong SH. Relativistic Treatment of Spinless Particles Subject to a Rotating Deng-Fan Oscillator. Commun Theor Phys. 2011;55(6):969-971. doi:10.108 8/0253-6102/55/6/05

46. Boonserm P, Visser M. Quasi-normal frequencies: key analytic results. J High Energ Phys. 2011;2011(3):73. doi:10.1007/ihep03(2011)073

47. Yazarloo BH, Hassanabadi H, Zarrinkamar S. Oscillator strengths based on the Möbius square potential under Schrödinger equation. Eur Phys J Plus. 2012;127(5):51. doi:10.1140/epjp/i2012-12051-9

48. Greene RL, Aldrich C. Variational wave functions for a screened Coulomb potential. Phys Rev A. 1976;14(6):2363-2366. doi:10.1103/physreva.14.2363

49. Abramowitz M, Stegun IA. Handbook of Mathematical Function with Formulas, Graphs, and Mathematical Tables. National Bureau of Standards Applied Mathematics Series-55; 1964. doi:10.1115/ 1.3625776

50. Maireche A. The Relativistic and Nonrelativistic Solutions for the Modified Unequal Mixture of Scalar and Time-Like Vector Cornell Potentials in the Symmetries of Noncommutative Quantum Mechanics. Jordan J Phys. 2021;14(1):59-70. doi:10.47 $\underline{011 / 14.1 .6}$

51. Maireche A. The Klein-Gordon equation with modified Coulomb plus inverse-square potential in the noncommutative three-dimensional space. $M o d$ Phys Lett A. 2020;35(5):052050015. doi:10.1142/s0217 $\underline{732320500157}$

52. Maireche A. A Theoretical Model of Deformed Klein-Gordon Equation with Generalized Modified Screened Coulomb Plus Inversely Quadratic Yukawa Potential in RNCQM Symmetries. Few-Body Syst. 2021;62(1):12. doi:10.1007/s00601-021-01596-2

53. Maireche A. Bound-state Solutions of KleinGordon and Schrödinger Equations for Arbitrary 1state with Linear Combination of Hulthén and Kratzer Potentials. Afr Rev. 2020;15(003):19-31.

54. Motavalli H, Akbarieh AR. Klein-Gordon equation for the Coulomb potential in noncommutative space. Mod Phys Lett A. 2010;25(29):2523-2528. doi:10.1142/ $\underline{\mathrm{s} 0217732310033529}$
55. Darroodi M, Mehraban H, Hassanabadi S. The Klein-Gordon equation with the Kratzer potential in the noncommutative space. Mod Phys Lett A. 2018;33(35):1850203. doi:10.1142/s021773231850203 $\underline{6}$

56. Maireche A. Modified Unequal Mixture Scalar Vector Hulthén-Yukawa Potentials Model as a Quark-Antiquark Interaction and Neutral Atoms via Relativistic Treatment Using the Improved Approximation of the Centrifugal Term and Bopp's Shift Method. Few-Body Syst. 2020;61(3):30. doi:10.10 07/s00601-020-01559-z

57. Mezincescu L. Star operation in quantum mechanics. E-print. 2000. arXiv: hep-th/0007046v2.

58. Bopp F. La mécanique quantique est-elle une mécanique statistique classique particulière? Ann Inst Henri Poincaré. 1956;15:81.

59. Gouba L. A comparative review of four formulations of noncommutative quantum mechanics. Int J Mod Phys A. 2016;31(19):1630025. do $\mathrm{i}: 10.1142 / \mathrm{s} 0217751 \times 16300258$

60. Maireche A. New nonrelativistic bound state solutions of the NGECSC potential involving GECSC potential and new central terms for hydrogen atom. Afr Rev Phys. 2019;13(0023):142-149. http://lamp.ict p.it/index.php/aphysrev/article/view/1635/590.

61. Maireche A. A theoretical investigation of nonrelativistic bound state solution at finite temperature using the sum of modified Cornell plus inverse quadratic potential. Sri Lankan J Phys. 2020;21(1):11-35. doi:10.4038/sljp.v21i1.8069

62. Maireche A. Modified Schrödinger Equation with Modified QM Gravitational and Harmonic Oscillator Potentials in Symmetries of Non-commutative QM. Afr Rev Phys. 2019;14(0018):130-139.

63. Maireche A. A Complete Analytical Solution of the Mie-Type Potentials in Non-commutative 3-Dimensional Spaces and Phases Symmetries. Afr Rev Phys. 2016;11(0015):111-117.

64. Maireche A. Nonrelativistic treatment of Hydrogen-like and neutral atoms subjected to the generalized perturbed Yukawa potential with centrifugal barrier in the symmetries of noncommutative Quantum mechanics. Int J Geom Methods Mod Phys. 2020;17(5):2050067. doi:10.1142/s 021988782050067x

65. Maireche A. Theoretical Investigation of the Modified Screened cosine Kratzer potential via Relativistic and Nonrelativistic treatment in the NCQM symmetries. Lat Am J Phys Educ. 2020;14(3):3310. 
66. Maireche A. Solutions of Klein-Gordon equation for the modified central complex potential in the symmetries of noncommutative quantum mechanics. Sri Lankan J Phys. 2021;22(1):1-19. doi:10.4038/sljp.v2 $\underline{2 i 1.8079}$

67. Maireche A. Investigations on the Relativistic Interactions in One-Electron Atoms with Modified Yukawa Potential for Spin 1/2 Particles. International Frontier Science Letters. 2017;11:29-44. doi:10.18052/ www.scipress.com/ifsl.11.29

68. Maireche A. New Relativistic Bound States for Modified Pseudoharmonic Potential of Dirac Equation with Spin and Pseudo-Spin Symmetry in Oneelectron Atoms. Afr Rev Phys. 2018;12(0018):130-143.

69. Maireche A. New Relativistic Atomic Mass Spectra of Quark ( $\mathrm{u}, \mathrm{d}$ and s) for Extended Modified Cornell Potential in Nano and Plank's Scales. J Nano- Electron Phys. 2016;8(1):01020. doi:10.21272/jnep.8(1).01020

70. Maireche A. A New Relativistic Study for Interactions in One-electron atoms (Spin $1 / 2$ Particles) with Modified Mie-type Potential. J Nano- Electron Phys. 2016;8(4):04027. doi:10.21272/jnep.8(4(1)).0402 $\underline{7}$

71. Saidi A, Sedra MB. Spin-one $(1+3)$-dimensional DKP equation with modified Kratzer potential in the non-commutative space. Mod Phys Lett A.

2019;35(5):2050014. doi:10.1142/s0217732320500145

72. Badawi M, Bessis N, Bessis G. On the introduction of the rotation-vibration coupling in diatomic molecules and the factorization method. J Phys B: At Mol Phys. 1972;5(8):L157-L159. doi:10.1088/0022-370 $\underline{0 / 5 / 8 / 004}$

73. Dong SH, Qiang WC, Sun GH, Bezerra VB. Analytical approximations to thel-wave solutions of the Schrödinger equation with the Eckart potential. J Phys A: Math Theor. 2007;40(34):10535-10540. doi:1 $\underline{0.1088 / 1751-8113 / 40 / 34 / 010}$
74. Zhang Y. Approximate analytical solutions of the Klein-Gordon equation with scalar and vector Eckart potentials. Phys Scr. 2008;78(1):015006. doi:10.1088/0 031-8949/78/01/015006

75. Bencheikh K, Medjedel S, Vignale G. Current reversals in rapidly rotating ultracold Fermi gases. Phys Rev A. 2014;89(6). doi:10.1103/physreva.89.0636 $\underline{20}$

76. Medjedel S, Bencheikh K. Exact analytical results for density profile in Fourier space and elastic scattering function of a rotating harmonically confined ultra-cold Fermi gas. Phys Lett A. 2019;383(16):1915-1921. doi:10.1016/i.physleta.201 $\underline{9.03 .021}$

77. Maghsoodi E, Hassanabadi H, Zarrinkamar S. Spectrum of Dirac Equation Under Deng-Fan Scalar and Vector Potentials and a Coulomb Tensor Interaction by SUSYQM. Few-Body Syst. 2012;53(3-4):525-538. doi:10.1007/s00601-012-031 $\underline{4-5}$

78. Gnatenko KP, Tkachuk VM. Upper bound on the momentum scale in noncommutative phase space of canonical type. Euro physics Letters. 2019;127(2):20008. doi:10.1209/0295-5075/127/20008

79. Gnatenko KP, Tkachuk VM. Composite system in rotationally invariant noncommutative phase space. Int J Mod Phys A. 2018;33(07):1850037. doi:10.1142/s0 $\underline{217751 \times 18500379}$

80. Gnatenko KP. Composite system in noncommutative space and the equivalence principle. Physics Letters A. 2013;377(43):3061-3066. doi:10.101 6/j.physleta.2013.09.036

81. Maireche A. Bound-state solutions of the modified Klein-Gordon and Schrödinger equations for arbitrary l-state with the modified Morse potential in the symmetries of noncommutative quantum mechanics. J Phys Stud. 2021;25(1):1002. doi:10.3097 0/ips.25.1002 
النموذج النسبي واللانسبي الجديد للجزيئات ثثائية الأرة والجسيمات الفيرميومبية المتفاعلة مع كمون موبيس المغير والمحسن في إطار تناظرات الميكانيك الكمي اللاتبادلي الأستاذ الدكتور: عبد المجيذ معيرش

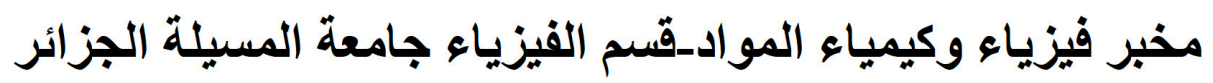

